\title{
Holographic approach to deformations of NS5-brane distributions and exact CFTs
}

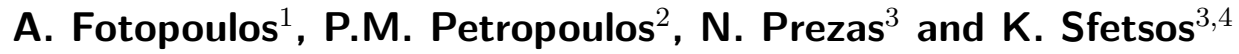 \\ 1 Dipartimento di Fisica Teorica dell'Università di Torino and INFN, \\ Sezione di Torino, via P. Giuria 1, 10125 Torino, Italy \\ Email: foto@to.infn.it \\ 2 Centre de Physique Théorique, Ecole Polytechnique, CNRS-UMR 7644, \\ 91128 Palaiseau Cedex, France \\ Email: marios@cpht.polytechnique.fr \\ 3 Theory Unit, Physics Department, CERN, \\ 1211 Geneva 23, Switzerland \\ Email: nikolaos.prezas@cern.ch \\ 4 Department of Engineering Sciences, University of Patras, \\ 26110 Patras, Greece \\ Email: sfetsos@upatras.gr
}

ABSTRACT: We consider general planar deformations of a circular distribution of NS5-branes. The near-horizon region of the latter admits, after a T-duality transformation, an exact conformal-field-theory description in terms of the coset model $S U(2) / U(1) \times S L(2, \mathbb{R}) / U(1)$. We derive the exactly marginal operators corresponding to an infinitesimal planar deformation using the conjectured holography between the coset model and the little string theory that resides on the worldvolume of the NS5-branes. Subsequently, we perform a complementary analysis of the same deformations using the associated $\mathcal{N}=1$ supersymmetric $\sigma$ model and verify the holographic correspondence. We explicitly demonstrate a precise match between the two approaches which rests upon a delicate interplay between exact conformalfield-theory operators and their semiclassical realizations in terms of target-space variables. 


\section{Contents}

1. Introduction 2

2. Neveu-Schwarz five-branes and exact conformal field theories 4

2.1 Neveu-Schwarz five-branes on a point and on a circle 4

2.2 Vertex operators for the coset theories 6

$\begin{array}{lll}2.3 & \text { Semiclassical geometry and parafermions } & 7\end{array}$

2.4 Semiclassical description of conformal primaries 9

3. Holographic approach to NS5-brane deformations 11

3.1 Holographic dictionary: generalities 12

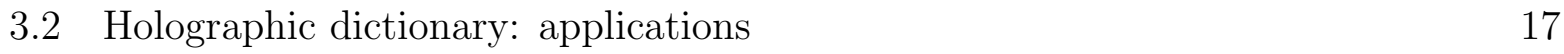

$\begin{array}{lll}3.2 .1 & \text { Point to circle } & 17\end{array}$

$\begin{array}{lll}3.2 .2 & \text { Circle to circle } & 18\end{array}$

3.2.3 Circle to ellipsis $\quad 19$

3.2.4 General circle deformations 21

4. Deformations of NS5-branes: $\sigma$ model approach and comparison 23

4.1 Neveu-Schwarz five-branes on an ellipsis 23

4.2 General deformations of circular NS5-brane distribution 28

5. Summary and perspectives $\quad 32$

A. Linear-dilaton background and the $\mathcal{N}=4$ superconformal algebra $\quad 34$

B. Parafermionic operator products $\quad 37$

B.1 Compact parafermions and $S U(2)$ current algebra $\quad 37$

B.2 Non-compact parafermions and $S L(2, \mathbb{R})$ current algebra 39

C. Superconformal $\mathcal{N}=2$ algebras $\quad 40$

D. Coframes, spin connections and curvature two-forms 41 


\section{Introduction}

Linear-dilaton backgrounds were recognized long ago as string vacua with rich properties and diverse applications [1,2]. An important step was taken in [3], where the connection between the linear-dilaton exact worldsheet theory and the solitonic target-space objects known as NS5-branes [4] was established. Distributions of NS5-branes in their transverse space generate exact string backgrounds with half supersymmetry broken. Situations where the underlying $\sigma$ model can be identified with a known exact conformal field theory (CFT) are especially desirable but nevertheless rare. When $k$ parallel NS5-branes are located at the same point, their transverse near-horizon geometry is the target space of the $\mathbb{R}_{\phi} \times S U(2)_{k}$ supersymmetric Wess-Zumino-Witten (WZW) model $-\mathbb{R}_{\phi}$ denotes the radial direction that supports the linear dilaton [3]. This background exhibits the $\mathcal{N}=4$ superconformal algebra and its string spectrum was analyzed in [5]. The only other known case resulting into an exact CFT description is when the NS5 branes are uniformly distributed on the circumference of a circle [6]. This is also a remarkable theory since the geometry, two-form and dilaton backgrounds turn out [6] to be T-dual to those of the $S U(2) / U(1) \times S L(2, \mathbb{R}) / U(1)$ product of conformal cosets.

Over the recent years, NS5-brane distributions attracted further attention in the advent of holography. In this framework, it is conjectured that string theory on linear-dilatonlike vacua is the holographic dual of little string theories (LSTs) [7]. The latter are nongravitational theories that capture the dynamics of the worldvolume modes of NS5-branes in some appropriate decoupling limit.

An important consequence of the above holographic duality is the correspondence between vertex operators of the string theory on the asymptotic linear-dilaton background and deformations of the dual string theory $[8,9]$. Hence, starting from a specific NS5-brane distribution, one can trigger perturbations by giving vacuum expectation values (VEVs) to appropriate scalar fields defined on their worldvolumes. Such perturbations amount to displacements of the NS5-branes in their four-dimensional transverse space, and their effect on the underlying two-dimensional worldsheet theory can be immediately uncovered using the holographic dictionary.

The present work aims primarily at demonstrating the validity of the above holographic dictionary in situations where the effect of the perturbations on the locus of the NS5-branes can be independently controlled at the level of the worldsheet theory. This is possible whenever the two-dimensional $\sigma$ model that describes the string dynamics is an exact and solvable CFT. The case that we will be dealing with falls in this class and provides the first example where the little string holographic duality is checked with accuracy.

Exact conformal $\sigma$ models serve to generate continuous families of exact string vacua using e.g. integrable marginal worldsheet operators. The expected interplay between perturbed worldsheet $\sigma$ models and deformed target-space distributions of source branes has been 
analyzed in several instances [10]-[15] based on a general criterion established in [16] for current-current perturbations. However, the first example of a clear relationship between a marginal worldsheet operator and a geometrical deformation of the NS5-brane density distribution has been worked out in [17]. There, it was shown that a continuous deformation of the circular NS5-brane distribution into an elliptic one was driven by a marginal perturbation of the $S U(2) / U(1) \times S L(2, \mathbb{R}) / U(1)$ worldsheet $\sigma$ model. The bonus of this analysis was to demonstrate that the compact parafermions of the $S U(2) / U(1)$ theory could be appropriately dressed by non-compact vertex operators of the $S L(2, \mathbb{R}) / U(1)$ coset and form a novel kind of operator with anomalous dimension two which is non-factorizable in terms of holomorphic and anti-holomorphic currents. This marginal operator was responsible for the circular distribution of NS5-branes being deformed into an elliptical one.

The deformation of the circle into an ellipsis may be thought of as one particular mode among an infinitude consisting of battered circles with $n \in \mathbb{N}$ bumps, distributed with $\mathbb{Z}_{n}$ symmetry around the original circle. Correspondingly, one may expect other combinations of compact parafermions with non-compact or even compact dressings to provide new dimension-two operators, each one triggering a mode with a given number of bumps in the associated NS5brane distribution. Another motivation for our article is to demonstrate this statement for the supersymmetric $S U(2) / U(1) \times S L(2, \mathbb{R}) / U(1)$ coset model. Not only this achievement is interesting per se as an original conformal-field-theoretical result, but it also establishes one of the sides of the sought after holographic correspondence that we advertised previously.

The strategy we will follow is:

- First, we analyze in detail the spectrum of conformal operators of the unperturbed theory. The latter is the near-horizon background created by $k$ parallel NS5-branes uniformly distributed on a circle which, after T-duality, is the product of two supersymmetric Kazama-Suzuki cosets, $S U(2) / U(1) \times S L(2, \mathbb{R}) / U(1)$, as mentioned above. These models have chiral and anti-chiral parafermions which are not currents since their conformal weights are smaller than one in the case of $S U(2) / U(1)$ or greater than one in the case of $S L(2, \mathbb{R}) / U(1)$. Nonetheless, compact parafermions can be successfully combined with non-compact and compact primaries to deliver dimension-two operators. For our analysis their necessary semiclassical expressions can be worked out using group-theory methods.

- Next, we move to the LST side and consider a class of worldvolume operators whose VEVs describe displacements of the NS5-branes. These operators must correspond to marginal worldsheet operators preserving the original $\mathcal{N}=4$ superconformal symmetry, like any transverse-space distribution of NS5-branes. Using the holographic dictionary, we can indeed associate to those LST operators the marginal operators of the $S U(2) / U(1) \times S L(2, \mathbb{R}) / U(1)$, built previously as compact parafermions appropriately dressed with conformal primaries from the non-compact as well as the compact coset in general. 
- Finally, we can independently check that the worldsheet marginal operators constructed by following the holographic recipe, do trigger the expected geometric displacements. Put differently, we must reinterpret the effect of these operators on the $\mathcal{N}=1$ supersymmetric $\sigma$-model background fields and check that these perturbed background fields (metric, spin connection, curvature two-form, antisymmetric tensor and dilaton) are indeed generated by a distribution of NS5-branes in conformity with the distribution predicted by the original LST pattern. To perform this comparison a delicate interplay between CFT operators and their semiclassical expressions takes place.

These three steps are taken in Secs. 2, 3 and 4, respectively. Put together, they demonstrate the validity of the holographic dictionary and establish the correspondence among marginal operators of the supersymmetric $S U(2) / U(1) \times S L(2, \mathbb{R}) / U(1)$ model and $n$-bump deformations of the circular distribution of NS5-branes. The main text is followed by several appendices, which provide the reader with all necessary computational details: compact and non-compact parafermionic fields and their operator product expansions (OPEs), $\mathcal{N}=4$ and $\mathcal{N}=2$ extended superconformal algebras, and finally general properties of the $n$-bumpdeformed geometries (coframes, spin connections and curvature two-forms).

\section{Neveu-Schwarz five-branes and exact conformal field theories}

In this section we first recall a few facts on the exact CFT description of the NS5-branes on a point or distributed uniformly over the circumference of a circle, which concerns, respectively, the $S U(2) \times \mathbb{R}_{\phi}$ or $S U(2) / U(1) \times S L(2, \mathbb{R}) / U(1)$ theories and the associated operators. Next we review material on the classical parafermions relevant for our paper and develop the semiclassical correspondence of CFT primary operators to explicit expressions in terms of target-space fields, which is crucial for the comparisons that we will perform in Sec. 4.

\subsection{Neveu-Schwarz five-branes on a point and on a circle}

A distribution of a large number $k$ of parallel NS5-branes with density $\rho(\mathbf{x})$ in the transverse

$\mathbb{R}^{4}$ space is described, to leading order in $\alpha^{\prime}$, as a supergravity background specified by a ten-dimensional metric of the form

$$
d s^{2}=\eta_{\mu \nu} d x^{\mu} d x^{\nu}+H(\mathbf{x}) \delta_{i j} d x^{i} d x^{j},
$$

where $\eta_{\mu \nu}$ is the Minkowski metric on the flat worldvolume of the NS5-branes parameterized by $x^{\mu}, \mu=0,1, \ldots, 5$ and $\mathbf{x}=\left\{x^{i}, i=6,7,8,9\right\}$ labels the space $\mathbb{R}^{4}$ transverse to the NS5branes. The geometry is accompanied by a three-form NS-NS field

$$
H_{i j k}=\epsilon_{i j k}{ }^{l} \partial_{l} H,
$$

where the indices are lowered and raised with the flat metric of $\mathbb{R}^{4}$, and by a dilaton field given by

$$
e^{2\left(\Phi-\Phi_{0}\right)}=H,
$$


where $\Phi_{0}$ is related to the asymptotic string coupling $g_{\mathrm{s}}=\exp 2 \Phi_{0}$ far from the NS5-branes. In general, the above background fields provide a solution of the supergravity equations of motion, which preserves one half of the maximum supersymmetry, if the function $H(\mathbf{x})$ is harmonic in $\mathbb{R}^{4}$. The function $H(\mathbf{x})$ is specified in terms of the density as

$$
H(\mathbf{x})=1+\alpha^{\prime} k \int_{\mathbb{R}^{4}} d^{4} x^{\prime} \frac{\rho\left(\mathbf{x}^{\prime}\right)}{\left|\mathbf{x}-\mathbf{x}^{\prime}\right|^{2}}
$$

Although it is believed that the above background can be promoted to a string solution valid to all orders in $\alpha^{\prime}$ for an arbitrary distribution of NS5-branes, the underlying exact conformal field theory is not known in general. There are, however, two special configurations whose near-horizon limit, corresponding to the harmonic function (2.4) with the 1 removed, admit a CFT description. The first is the case of NS5-branes put at the same point $\mathbf{x}=\mathbf{0}[3]$. The corresponding harmonic function is $H(\mathbf{x})=k / r^{2}$, with $r$ being the radial distance in $\mathbb{R}^{4}$ and after a reparameterization $r=\sqrt{\alpha^{\prime} k} \exp \left(\Phi_{0}+\phi / \sqrt{\alpha^{\prime} k}\right)$, the metric and the three-form become

$$
d s^{2}=d s^{2}\left(E^{(1,5)}\right)+d \phi^{2}+\alpha^{\prime} k d \Omega_{3}^{2}, \quad H=2 \mathrm{Vol}_{S^{3}},
$$

where $d \Omega_{3}^{2}$ is the line element of the transverse $S^{3}$ and $\mathrm{Vol}_{S^{3}}$ is its volume form. The dilaton is linear in $\phi$

$$
\Phi=-\frac{q}{2} \phi, \quad q=\frac{2}{\sqrt{\alpha^{\prime} k}} .
$$

The three-sphere of radius $\sqrt{\alpha^{\prime} k}$ along with the NS-NS flux can be described by an $S U(2)$ Wess-Zumino-Witten (WZW) model at level $k$, while the linear dilaton corresponds to a free boson with background charge $q$. Hence, the near-horizon region of a system of parallel and coincident NS5-branes admits an exact conformal field theory description in terms of the Callan-Harvey-Strominger (CHS) background

$$
\mathbb{R}^{5,1} \times \mathbb{R}_{\phi} \times S U(2)_{k}
$$

The supersymmetric $S U(2)_{k}$ WZW model consists of a bosonic $S U(2)$ WZW model at level $k-2$, whose affine primaries $\Phi_{j ; m, \bar{m}}^{s u}$ have conformal weight

$$
h=\frac{j(j+1)}{k}
$$

and three free fermions $\psi_{a}, a=1,2,3$ transforming in the adjoint representation of $S U(2)$. The conformal primaries of $\mathbb{R}_{\phi}$ are $e^{a \phi}$ and their dimension is

$$
h=-\frac{1}{2} a(a+q) .
$$

We have also the worldsheet superpartners of $x^{\mu}$ and $\phi$ given by free fermions $\psi_{\mu}$ and $\psi_{\phi}$. The background $\mathbb{R}_{\phi} \times S U(2)_{k}$ supports the small $\mathcal{N}=4$ superconformal algebra [5] and in 
App. A we present for reference the relevant details. The central charges of the three CFT factors are

$$
\begin{aligned}
c_{5,1} & =6+\frac{6}{2}, \\
c_{\phi} & =1+\frac{3 \alpha^{\prime}}{2} q^{2}+\frac{1}{2}, \\
c_{k} & =\frac{3(k-2)}{k}+\frac{3}{2} .
\end{aligned}
$$

They add up to $c=15$, as they should in order to have a vanishing total conformal anomaly. In the rest of this paper $\alpha^{\prime}$ is set to 2 .

The conformal field theory background (2.7) suffers from a singularity at $\phi=-\infty$ where the string coupling diverges. Perturbation theory breaks down in this region and hence (2.7) is a good description of the physics only far from the NS5-branes. The strong-coupling singularity is due to the fact that the NS5-branes are coincident since a single NS5-brane does not develop the "throat" geometry that results in the linear dilaton. Hence, separating the NS5-branes should cure the strong-coupling singularity. At the same time, however, we would like to keep the benefits of an exact conformal field theory description. The only known configuration that achieves that, is a continuous and uniform distribution of NS5branes on the circumference of a circle found in [6], where it was shown that in this case the non-trivial part of the CHS background (2.7) is replaced, after an appropriate T-duality, by the product of two Kazama-Suzuki coset models

$$
\frac{S U(2)_{k}}{U(1)} \times \frac{S L(2, \mathbb{R})_{k}}{U(1)}
$$

orbifolded under a $\mathbb{Z}_{k}$ discrete symmetry. Actually, as we will see soon, (2.11) arises as an exactly marginal deformation of the CHS background.

\subsection{Vertex operators for the coset theories}

The first factor in (2.11) is the $\mathcal{N}=2$ minimal model at level $k$. We will denote its NS-NS sector primaries by $V_{j ; m, \bar{m}}^{s u}$. Their conformal weight and $R$-charge in the holomorphic sector are

$$
h=\frac{j(j+1)-m^{2}}{k}, \quad \mathcal{Q}_{\mathrm{R}}=-\frac{2 m}{k} .
$$

Similar formulas apply for the antiholomorphic sector with $m$ replaced by $\bar{m}$. A very useful representation of the minimal model is in terms of a bosonic coset $S U(2)_{k-2} / U(1)$, i.e. the compact parafermion theory, and a compact canonically normalized free boson $P[18]$. The latter bosonizes the two free fermions that, along with the bosonic $S U(2)_{k-2} / U(1)$ coset, realize the supersymmetric $S U(2)_{k} / U(1)$ Kazama-Suzuki model. The operator $V_{j ; m, \bar{m}}^{s u}$ is decomposed as

$$
V_{j ; m, \bar{m}}^{s u}=\psi_{j ; m, \bar{m}} \exp \left(i \frac{2 m}{\sqrt{k(k-2)}} P_{\mathrm{L}}+i \frac{2 \bar{m}}{\sqrt{k(k-2)}} P_{\mathrm{R}}\right)
$$


where $\psi_{j ; m, \bar{m}}$ are primaries of the parafermion theory [19] at level $k-2$ and $P_{\mathrm{L}}(z), P_{\mathrm{R}}(\bar{z})$ are the holomorphic and antiholomorphic parts of $P$. The conformal dimension of $\psi_{j ; m, \bar{m}}$ is

$$
h=\frac{j(j+1)}{k}-\frac{m^{2}}{k-2}
$$

and upon adding to it $\frac{2 m^{2}}{k(k-2)}$, i.e. the conformal dimension of the exponential, we obtain the superconformal weight in (2.12). An interesting property of the parafermion theory that we will use is the equivalence of primaries [19]

$$
\psi_{j ; m, \bar{m}} \equiv \psi_{\frac{k-2}{2}-j ;-\frac{k-2}{2}+m,-\frac{k-2}{2}+\bar{m}} .
$$

This equivalence relates primaries with $-j \leqslant m \leqslant j$ originating from $S U(2)_{k-2}$ affine primaries, to primaries with $j \leqslant m \leqslant k-2-j$. For the latter the conformal dimension is given by (2.14) with the term $m-j$ added on the right-hand side.

The second factor in (2.11) is the Kazama-Suzuki model based on the $S L(2, \mathbb{R}) / U(1)$ noncompact coset. Its NS-NS primaries $V_{j ; m, \bar{m}}^{s l}$ have conformal weight and $R$-charge given by

$$
h=\frac{-j(j+1)+m^{2}}{k}, \quad \mathcal{Q}_{\mathrm{R}}=\frac{2 m}{k} .
$$

As for the $\mathcal{N}=2$ minimal model, there is a useful representation of this Kazama-Suzuki model in terms of the non-compact parafermion theory $S L(2, \mathbb{R})_{k+2} / U(1)[20]$ and a free scalar $Q$. The vertex operators $V_{j ; m, \bar{m}}^{s l}$ decompose as

$$
V_{j ; m, \bar{m}}^{s l}=\pi_{j ; m, \bar{m}} \exp \left(i \frac{2 m}{\sqrt{k(k+2)}} Q_{\mathrm{L}}+i \frac{2 \bar{m}}{\sqrt{k(k+2)}} Q_{\mathrm{R}}\right),
$$

where $\pi_{j ; m, \bar{m}}$ are primaries of the non-compact parafermion theory at level $k+2$, and $Q_{\mathrm{L}}(z)$ and $Q_{\mathrm{R}}(\bar{z})$ are the holomorphic and antiholomorphic parts of $Q$. The conformal dimensions of $\pi_{j ; m, \bar{m}}$ read:

$$
h=-\frac{j(j+1)}{k}+\frac{m^{2}}{k+2}
$$

and, along with the contribution $\frac{2 m^{2}}{k(k+2)}$ of the exponential, add up to the superconformal weight given in Eq. (2.16). As in the compact case, there is an equivalence between noncompact parafermion primaries

$$
\pi_{j ; m, \bar{m}}=\pi_{\frac{k-2}{2}-j ; \frac{k+2}{2}+m, \frac{k+2}{2}+\bar{m}} .
$$

\subsection{Semiclassical geometry and parafermions}

The coset $S U(2)_{k-2} / U(1)$ has natural chirally and anti-chirally conserved objects $\psi, \psi^{\dagger}$ and $\bar{\psi}, \bar{\psi}^{\dagger}$ respectively, known as parafermions, with conformal dimensions [19]

$$
h=1-\frac{1}{k-2}, \quad \bar{h}=1-\frac{1}{k-2} .
$$


The parafermion theory, being a coset CFT, it admits a description as a gauged WZW model [21]. Semiclassically, the latter yields a $\sigma$ model with a bell-like target-space geometry [22] and a varying dilaton [23]

$$
d s_{S U(2) / U(1)}^{2}=k\left(d \theta^{2}+\tan ^{2} \theta d \varphi^{2}\right), \quad e^{-2 \Phi}=\cos ^{2} \theta
$$

with $\theta \in[0, \pi], \varphi \in[0,2 \pi)$ and $\varphi \equiv \varphi+\frac{2 \pi}{k}$. In the standard parafermion theory the compact scalar $\varphi$ has period $2 \pi$ but here we have changed its period to $2 \pi / k$ so that it corresponds to the $\mathbb{Z}_{k}$ orbifold of the original parafermion theory. In terms of the $\sigma$-model variables, the classical parafermion fields read [22]:

$$
\psi=(\partial \theta-i \tan \theta \partial \varphi) e^{-i\left(\varphi+\phi_{1}\right)}, \quad \psi^{\dagger}=(\partial \theta+i \tan \theta \partial \varphi) e^{i\left(\varphi+\phi_{1}\right)}
$$

and

$$
\bar{\psi}=(\bar{\partial} \theta-i \tan \theta \bar{\partial} \varphi) e^{-i\left(\varphi-\phi_{1}\right)}, \quad \bar{\psi}^{\dagger}=(\bar{\partial} \theta+i \tan \theta \bar{\partial} \varphi) e^{i\left(\varphi-\phi_{1}\right)} .
$$

The overall normalization is chosen such that the OPE of the corresponding quantum parafermions in App. B agree with the Poisson brackets of their above classical counterparts. The parafermions have their origin in the currents $J^{ \pm}$and $\bar{J}^{ \pm}$of the $S U(2)_{k}$ theory. In the gauged theory they are dressed with gauge fields that render them gauge-invariant. That explains also the presence of the phase $\phi_{1}$ which is a non-local function of the variables $\theta$ and $\varphi$. Its explicit expression is not needed here (see, for instance, [17]), but it is necessary for ensuring on-shell conservation of the parafermions

$$
\bar{\partial} \psi=\bar{\partial} \psi^{\dagger}=0, \quad \partial \bar{\psi}=\partial \bar{\psi}^{\dagger}=0 .
$$

The non-local phase $\phi_{1}$ should drop out in expressions having a clear local field theory interpetation, for instance those appearing in the two-dimensional $\sigma$ model actions as we shall see.

The non-compact coset $S L(2, \mathbb{R})_{k+2} / U(1)$ has also natural chirally and anti-chirally conserved objects $\pi, \pi^{\dagger}$ and $\bar{\pi}, \bar{\pi}^{\dagger}$ respectively, known as non-compact parafermions, with conformal dimensions [20]

$$
h=1+\frac{1}{k+2}, \quad \bar{h}=1+\frac{1}{k+2} .
$$

The non-compact-parafermion theory admits a semiclassical description in terms of a $\sigma$ model with either a cigar-shaped or a trumpet-shaped geometry along with a non-trivial dilaton [23]. We will consider the trumpet picture, specified by

$$
d s_{S L(2, \mathbb{R}) / U(1)}^{2}=k\left(d \rho^{2}+\operatorname{coth}^{2} \rho d \omega^{2}\right), \quad e^{-2 \Phi}=\sinh ^{2} \rho,
$$

with coordinates $\rho \in[0, \infty), \omega \in[0,2 \pi)$ and $\omega \equiv \omega+\frac{2 \pi}{k}$. This metric is singular near $\rho=0$ but its T-dual, namely the cigar, is well-defined and provides an equivalent (up to T-duality) semiclassical description of the non-compact parafermion theory. The classical non-compact parafermion fields are

$$
\pi=(\partial \rho+i \operatorname{coth} \rho \partial \omega) e^{i\left(\omega+\phi_{2}\right)}, \quad \pi^{\dagger}=(\partial \rho-i \operatorname{coth} \rho \partial \omega) e^{-i\left(\omega+\phi_{2}\right)}
$$


and

$$
\bar{\pi}=(\bar{\partial} \rho+i \operatorname{coth} \rho \bar{\partial} \omega) e^{i\left(\omega-\phi_{2}\right)}, \quad \bar{\pi}^{\dagger}=(\bar{\partial} \rho-i \operatorname{coth} \rho \bar{\partial} \omega) e^{-i\left(\omega-\phi_{2}\right)} .
$$

The phase $\phi_{2}$ is non-local and ensures on-shell conservation laws similar to those of the compact case, Eq. (2.24).

The full conformal field theory (2.11) corresponds semiclassically to a $\sigma$ model with metric and dilaton given by

$$
d s^{2}=k\left(d \theta^{2}+\tan ^{2} \theta d \varphi^{2}+d \rho^{2}+\operatorname{coth}^{2} \rho d \omega^{2}\right), \quad e^{-2 \Phi}=\cos ^{2} \theta \sinh ^{2} \rho .
$$

The relation of this background to that of NS5-branes distributed uniformly over a circle, follows explicitly by first changing coordinates as $\varphi=\tau$ and $\omega=\tau+\psi$ and then performing a T-duality transformation with respect to $\tau$ [6]. The focal point of this paper will be the interplay between the description of deformations of the circular distribution of NS5-branes in the $\sigma$-model language and the corresponding operators in the exact conformal field theory description. For that, an important ingredient will be the semiclassical expressions of the conformal primary fields of the theory (2.11), to the description of which we now turn.

\subsection{Semiclassical description of conformal primaries}

Conformal primaries in (2.11) are products of primaries of each factor. First we consider the semiclassical description of the primaries of the WZW model for the non-compact group $S L(2, \mathbb{R})$ from which the semiclassical primaries for the non-compact coset $S L(2, \mathbb{R}) / U(1)$ follow by rendering them gauge-invariant. These primaries are built up using the group element $^{1}$

$$
\left(\begin{array}{l}
g_{++} g_{+-} \\
g_{-+} g_{--}
\end{array}\right)=\left(\begin{array}{cc}
\cosh \rho e^{i\left(\theta_{\mathrm{L}}+\theta_{\mathrm{R}}\right) / 2} & \sinh \rho e^{-i\left(\theta_{\mathrm{L}}-\theta_{\mathrm{R}}\right) / 2} \\
\sinh \rho e^{i\left(\theta_{\mathrm{L}}-\theta_{\mathrm{R}}\right) / 2} & \cosh \rho e^{-i\left(\theta_{\mathrm{L}}+\theta_{\mathrm{R}}\right) / 2}
\end{array}\right)
$$

and they transform in the $\left(\frac{1}{2}, \frac{1}{2}\right)$ representation of $S L(2, \mathbb{R})_{\mathrm{L}} \times S L(2, \mathbb{R})_{\mathrm{R}}$ with $U(1)$ charges $\left( \pm \frac{1}{2}, \pm \frac{1}{2}\right)$, in all four combinations, in accordance with their index. The explicit transformation rules referring to $S L(2, \mathbb{R})_{\mathrm{L}}$ are

$$
\begin{aligned}
& \delta_{0} g_{ \pm \pm}=\mp \frac{i}{2} g_{ \pm \pm}, \quad \delta_{0} g_{ \pm \mp}=\mp \frac{i}{2} g_{ \pm \mp}, \\
& \delta_{-} g_{++}=i g_{-+}, \quad \delta_{+} g_{++}=0, \\
& \delta_{-} g_{+-}=i g_{--}, \quad \delta_{+} g_{+-}=0 \text {, } \\
& \delta_{-g_{-+}}=0, \quad \delta_{+} g_{-+}=-i g_{++} \text {, } \\
& \delta_{-} g_{--}=0, \quad \delta_{+} g_{--}=-i g_{+-},
\end{aligned}
$$

and act only on the first index of the group elements. The similar transformations with respect to $S L(2, \mathbb{R})_{\mathrm{R}}$ acting on the second index of the group elements, may have the same

\footnotetext{
${ }^{1}$ In our presentation we follow and extend the brief discussion in [17]. For an extensive overview and general expressions see [24] and also [25], where some of the primaries have been used in relation to the physics of the two-dimensional black hole.
} 
or the opposite signs as compared to those in (2.31) since the two transformations are unrelated. We choose the opposite sign since in the non-compact coset model we will gauge the vectorial $U(1)$ subgroup instead of the axial, as we will shortly discuss.

Being finite-dimensional, the above is not a unitary representation of $S L(2, \mathbb{R})_{\mathrm{L}} \times S L(2, \mathbb{R})_{\mathrm{R}}$, but we may construct other irreducible representations that are unitary by appropriate multiplications and inversions of the above group elements. In particular, for the positive and negative discrete series, for given spin $j, m$ takes the values $\pm(j+1, j+2, \ldots)$. In order for the semiclassical description to remain valid, we will assume $j \ll k$. Then it is obvious that the following expressions for the semiclassical primaries are unique

$$
\begin{aligned}
\pi_{j ; j+1, j+1} & =\frac{1}{g_{--}^{2(j+1)}}, & \pi_{j ;-j-1,-j-1} & =\frac{1}{g_{++}^{2(j+1)}}, \\
\pi_{j ; j+1,-j-1} & =\frac{1}{g_{-+}^{2(j+1)}}, & \pi_{j ;-j-1, j+1} & =\frac{1}{g_{+-}^{2(j+1)}},
\end{aligned}
$$

since they represent highest- or lowest-weight states. The other members of the representation are obtained by transforming appropriately the states in (2.32) with $\delta_{ \pm}$using (2.31). It is crucial for the precise comparison that we perform in the next section to have an agreement between normalization factors of various operators in the semiclassical and the exact CFT approaches. Hence we will normalize them according to (B.17) as

$$
\pi_{j ; m \pm 1, \bar{m}}=\frac{1}{m \pm(j+1)} \delta_{ \pm} \pi_{j ; m, \bar{m}}
$$

for the left as well as for the right $S U(2)$ transformations. Here we present only the expressions one needs in this paper

$$
\pi_{j ; j+2, j+1}=i \frac{g_{+-}}{g_{--}^{2 j+3}}, \quad \pi_{j ; j+1, j+2}=-i \frac{g_{-+}}{g_{--}^{2 j+3}}, \quad \pi_{j ; j+2, j+2}=\frac{2(j+1) g_{+-} g_{-+}-1}{2(j+1) g_{--}^{2 j+4}} .
$$

For the parafermionic coset theory, corresponding to the gauging of $S L(2, \mathbb{R})$ with respect to the vector $U(1)$ subgroup described semiclassicaly in terms of (2.26), we have the transformation $\delta \theta_{\mathrm{L}}=-\epsilon$ and $\delta \theta_{\mathrm{R}}=\epsilon$, so that the appropriate unitary gauge fixing is $\theta_{\mathrm{L}}=\theta_{\mathrm{R}}=\omega{ }^{2}$ The elements $g_{ \pm \pm}$are gauge-invariant, whereas $g_{ \pm \mp}$ are not. Those become gauge-invariant provided they are multiplied by the non-local phase factor $\phi_{2}$ that appears in the non-compact parafermions. The gauge-invariant group elements that should be used in (2.32) and (2.34) to construct the expressions for the semiclassical primaries of the parafermionic theory are thus

$$
\left.g_{ \pm \pm}\right|_{\text {g. }- \text { inv. }}=\cosh \rho e^{ \pm i \omega},\left.\quad g_{ \pm \mp}\right|_{\text {g. }- \text { inv. }}=\sinh \rho e^{ \pm i \phi_{2}}
$$

\footnotetext{
${ }^{2}$ The vector transformation is consistent with the sign difference between the left and right $S U(2)$ transformations, mentioned below (2.31). Indeed, since $i\left(\theta_{\mathrm{L}}+\theta_{\mathrm{R}}\right)=\ln \left(g_{++} / g_{--}\right)$and $i\left(\theta_{\mathrm{L}}-\theta_{\mathrm{R}}\right)=\ln \left(g_{-+} / g_{+-}\right)$, we can easily see that for the left (right) transformations with the $U(1)$ subgroup one obtains $\delta \theta_{\mathrm{R}}=\epsilon_{\mathrm{L}}$ and $\delta \theta_{\mathrm{L}}=0\left(\delta \theta_{\mathrm{R}}=0\right.$ and $\left.\delta \theta_{\mathrm{L}}=\epsilon_{\mathrm{R}}\right)$. Since by definition a vector transformation has $\epsilon_{\mathrm{L}}=-\epsilon_{\mathrm{R}}$, we see that this is indeed consistent with the transformations $\delta \theta_{\mathrm{L}}=-\epsilon=-\delta \theta_{\mathrm{R}}$.
} 
Form now on we drop the indicated index, keeping in mind that the associated semiclassical primaries correspond to the parafermionic coset theory. In the semiclassical correspondence that we will establish we will take into account the leading $1 / k$-correction to their classical dimension which is zero. Therefore the dimension of the above semiclassical primaries of the parafermionic theory (2.18), becomes

$$
h_{j ; j+\ell}=h_{j ;-j-\ell}=\frac{(2 \ell-1) j+\ell^{2}}{k}+\mathcal{O}\left(1 / k^{2}\right),
$$

accordingly for the left or the right factor and where $\ell=1,2$ in our case.

For the case of the semiclassical primaries of the compact coset $S U(2) / U(1)$ the procedure is quite similar. We will be brief since the expressions we need in this paper are fewer than those needed from the non-compact coset. The semiclassical $S U(2)$ compact primaries are built up using the group element (we will use tildes so that there is no confusion with the $S L(2, \mathbb{R})$ group element we used above)

$$
\left(\begin{array}{l}
\tilde{g}_{++} \\
\tilde{g}_{+-} \\
\tilde{g}_{-+} \\
\tilde{g}_{--}
\end{array}\right)=\left(\begin{array}{cc}
\cos \theta e^{i\left(\theta_{\mathrm{L}}+\theta_{\mathrm{R}}\right) / 2} & \sin \theta e^{-i\left(\theta_{\mathrm{L}}-\theta_{\mathrm{R}}\right) / 2} \\
-\sin \theta e^{i\left(\theta_{\mathrm{L}}-\theta_{\mathrm{R}}\right) / 2} & \cos \theta e^{-i\left(\theta_{\mathrm{L}}+\theta_{\mathrm{R}}\right) / 2}
\end{array}\right) .
$$

These transform similarly to $(2.31)$ and form the $\left(\frac{1}{2}, \frac{1}{2}\right)$ unitary representation of $S U(2)_{\mathrm{L}} \times$ $S U(2)_{\mathrm{R}}$ with $U(1)$ charges $\left( \pm \frac{1}{2}, \pm \frac{1}{2}\right)$, in all four combinations and in accordance with their index. In the present paper we will need only the expressions for

$$
\psi_{j ; j, j}=\tilde{g}_{++}^{2 j}, \quad \psi_{j ;-j,-j}=\tilde{g}_{--}^{2 j}, \quad \psi_{j ; j,-j}=\tilde{g}_{+-}^{2 j}, \quad \psi_{j ;-j, j}=\tilde{g}_{-+}^{2 j},
$$

which, for fixed $j$, are highest- or lowest-weight representations for the left and right $S U(2)$ factors. For the parafermionic $S U(2) / U(1)$ coset theory with axial gauging, corresponding to the background (2.21), we have the transformation $\delta \theta_{\mathrm{L}}=\epsilon$ and $\delta \theta_{\mathrm{R}}=\epsilon$, so that the appropriate unitary gauge fixing is $\theta_{\mathrm{L}}=-\theta_{\mathrm{R}}=\varphi$. The elements $g_{ \pm \mp}$ are gauge-invariant, whereas $g_{ \pm \pm}$are not. As before, it turns out that they become gauge-invariant when they are multiplied by the non-local phase factor that appears in the compact parafermions. Hence

$$
\left.\tilde{g}_{ \pm \mp}\right|_{\text {g. }- \text { inv. }}= \pm \sin \theta e^{\mp i \varphi},\left.\quad \tilde{g}_{ \pm \pm}\right|_{\text {g. }- \text { inv. }}=\cos \theta e^{\mp i \phi_{1}}
$$

The dimensions of the above semiclassical primaries (2.14) in the parafermionic theory, up to the order we are interested in, are

$$
h_{j ; j}=h_{j ;-j}=\frac{j}{k}+\mathcal{O}\left(1 / k^{2}\right)
$$

accordingly for the left or the right factor.

\section{Holographic approach to NS5-brane deformations}

In this section, after reviewing the procedure by which the theory is deformed based on the holographic conjecture, we compute explicitly the operators corresponding to our cases. 
Subsequently we find their semiclassical expressions in terms of target-space fields. Then we specialize our findings to some simple cases like the deformation of a point distribution into a circular one and that of a circular into one of a different radius. We then pay particular attention to the deformation of a circular into a an elliptical distribution which captures most of the essential points of our construction. Finally, we apply our results to the case of a general distribution which, compared to the elliptical one, presents some new features such as the appearance of composite operators.

\subsection{Holographic dictionary: generalities}

An interesting feature of both (2.7) and (2.11) is that asymptotically (i.e. large $\rho$ for (2.11) in terms of its $\sigma$-model description (2.29)), they are linear dilaton space-times. It has been proposed that string theory on such space-times provides a holographic description of the mysterious non-gravitational string theory, known as little string theory, that lives on the worldvolume of NS5-branes in the decoupling limit where the asymptotic string coupling is taken to zero [7]. This correspondence is very similar to the usual AdS/CFT duality since it relates the decoupled theory on a stack of branes with the supergravity or string theory on the near-horizon geometry induced by the branes [26, 27, 28].

The spectrum of states in backgrounds that asymptote to a linear dilaton falls into three classes with distinct physical significance. For instance in the CHS background, there are delta-function normalizable states whose vertex operators behave for large $\phi$ as $e^{\left(-\frac{q}{2}+i \lambda\right) \phi}$ with real $\lambda$. They describe incoming and outcoming waves carrying momentum $\lambda$ along the holographic direction $\phi$. Besides these states, the theory contains also normalizable states that decay rapidly as $\phi \rightarrow \infty$. Hence, they are supported in the strong-coupling region of large negative $\phi$ and they can be thought of as bound states associated with the NS5-branes. Finally, there exist non-normalizable states whose wavefunctions diverge at the weakly-coupled boundary $\phi \rightarrow \infty$.

The holographic duality conjectures a correspondence between vertex operators of the string theory on the asymptotic linear dilaton background and deformations of the dual little string theory $[8,9]$. More precisely, adding to the worldsheet Lagrangian a non-normalizable operator $V_{\text {non-nor. }}$ corresponds to perturbing the Lagrangian of the dual theory with an appropriate dual gauge-invariant operator $W_{V}$. If, instead, we add to the worldsheet theory the normalizable version $V_{\text {nor. }}$ of the same vertex operator, the dual theory does not change but the dual operator $W_{V}$ acquires a VEV $\left\langle W_{V}\right\rangle$ [29]. Since the geometry of the NS5-branes and their deformations are encoded in the VEVs of the adjoint scalar fields living on their worldvolume, we see that by employing the holographic correspondence we can uncover the associated deformations of the underlying (dual) CFT.

In the little string theory side, a basic class of operators we would like to consider and which encode all the information on the arrangement of the NS5-branes in their transverse $\mathbb{R}^{4}$ is given by chiral and gauge-invariant combinations of the adjoint scalar fields $\Phi^{i}, i=6,7,8,9$. The eigenvalues of these fields parameterize the positions of the NS5-branes in the four 
transverse directions, i.e. the moduli space of vacua of the little string theory. The operators of interest are $\operatorname{tr}\left(\Phi^{i_{1}} \Phi^{i_{2}} \ldots \Phi^{i_{2 j+2}}\right)$ with $2 j=0,1, \ldots,(k-2)$ and where we keep only the symmetric and traceless components in the indices $\left(i_{1}, i_{2}, \ldots, i_{2 j+2}\right)$ so that the operator is in a short representation of the supersymmetry algebra. ${ }^{3}$

The dictionary established in $[8,9]$ is

$$
\operatorname{tr}\left(\Phi^{i_{1}} \Phi^{i_{2}} \cdots \Phi^{i_{2 j+2}}\right) \leftrightarrow e^{-\varphi-\bar{\varphi}}\left(\psi \bar{\psi} \Phi_{j}^{s u}\right)_{j+1 ; m, \bar{m}} e^{-q(j+1) \phi},
$$

where the right-hand side refers to operators in the CHS background. We use the normalizable version of the CFT operators since we are interested in describing VEVs in the little string theory. We denoted by $\varphi, \bar{\varphi}$ the bosonized superconformal ghosts (which should not be confused with the compact coordinate of the bell geometry $(2.21)), \Phi_{j}^{s u}$ is an affine primary of the bosonic $S U(2)_{k-2}$ WZW model and the notation $\left(\psi \bar{\psi} \Phi_{j}^{s u}\right)_{j+1 ; m, \bar{m}}$ means that we should couple the fermions $\psi^{a}, a=3, \pm$ in the adjoint of $S U(2)$ with the bosonic primary in a primary of total spin $j+1$ and $\left(J_{3}^{\text {tot }}, \bar{J}_{3}^{\text {tot }}\right)=(m, \bar{m})$. We refer the reader to the App. A for further details on the notation. The values of $m$ and $\bar{m}$ are determined by the indices appearing at the left.

When the operators $\operatorname{tr}\left(\Phi^{i_{1}} \Phi^{i_{2}} \ldots \Phi^{i_{2 j+2}}\right)$ acquire non-zero VEVs, the Lagrangian of the dual worldsheet conformal field theory is perturbed as

$$
\mathcal{L}=\mathcal{L}_{0}+\left(\lambda_{j ; m, \bar{m}} G_{-\frac{1}{2}} \bar{G}_{-\frac{1}{2}}\left(\psi \bar{\psi} \Phi_{j}^{s u}\right)_{j+1 ; m, \bar{m}} e^{-q(j+1) \phi}+\text { c.c. }\right),
$$

where we have omitted the bosonized ghosts since we will be working in the 0-picture. These worldsheet deformations are marginal since $\left(\psi \bar{\psi} \Phi_{j}^{s u}\right)_{j+1 ; m, \bar{m}} e^{-q(j+1) \phi}$ has conformal weights $(h, \bar{h})=(1 / 2,1 / 2)$. Furthermore, they should leave unbroken the $\mathcal{N}=4$ superconformal symmetry of the original worldsheet theory since any configuration of parallel NS5-branes with arbitrary transverse positions preserves one-half of the maximum space-time supersymmetry. We check that $\mathcal{N}=4$ is indeed preserved in App. A. The couplings $\lambda_{j ; m, \bar{m}}$ are specified in terms of the VEVs of the LST operators while the supersymmetry generators $G_{-\frac{1}{2}}$ and $\bar{G}_{-\frac{1}{2}}$ correspond to the supercurrent $G$ defined in App. A. Notice that the perturbations we add in the worldsheet theory dominate at the region of strong coupling $\phi \rightarrow-\infty$ and provide a worldsheet potential that regularizes the strong-coupling singularity.

We will be interested in planar configurations of NS5-branes, i.e. distributions on the transverse plane $x^{8}-x^{9}$. It is very convenient then, following $[8,9]$, to use a parameterization of the moduli space in terms of two complex variables that span the two orthogonal hyperplanes transverse to the NS5-branes:

$$
A \equiv \Phi^{6}+i \Phi^{7}, \quad B \equiv \Phi^{8}+i \Phi^{9} .
$$

\footnotetext{
${ }^{3}$ There is also a subtlety pertaining to the precise definition of the trace. In principle, one should consider the usual single-trace along with multi-trace operators. However, for $j \ll k$, which will be the regime of our interest, the multi-trace contributions will be negligible and it will suffice to consider the single-trace ones $[8,9]$.
} 
Embedding the rotational $S O(2)_{A} \times S O(2)_{B}$ of the $A$ and $B$ planes in the $S U(2)_{\mathrm{L}} \times S U(2)_{\mathrm{R}}$ symmetry of the CHS background so that $S O(2)_{A}$ is generated by $J_{3}^{\text {tot }}-\bar{J}_{3}^{\text {tot }}$ and $S O(2)_{B}$ is generated by $J_{3}^{\text {tot }}+\bar{J}_{3}^{\text {tot }}$, yields the following charge assignments

$$
m_{A}=\frac{1}{2}, \quad \bar{m}_{A}=-\frac{1}{2}, \quad m_{B}=\frac{1}{2}, \quad \bar{m}_{B}=\frac{1}{2} .
$$

Combining those with the general relation (3.1) leads to the following correspondences

$$
\operatorname{tr}\left(A^{l} B^{2 j+2-l}\right) \leftrightarrow e^{-\varphi-\bar{\varphi}}\left(\psi \bar{\psi} \Phi_{j}^{s u}\right)_{j+1 ; j+1, j+1-l} e^{-q(j+1) \phi}
$$

and

$$
\operatorname{tr}\left(A^{l}\left(B^{*}\right)^{2 j+2-l}\right) \leftrightarrow e^{-\varphi-\bar{\varphi}}\left(\psi \bar{\psi} \Phi_{j}^{s u}\right)_{j+1 ;-j-1+l,-j-1} e^{-q(j+1) \phi} .
$$

We set $\langle A\rangle=0$ since we will study NS5-branes distributed on the $B$ plane and fixed at $x^{6}=x^{7}=0$. Their positions are parameterized by $k$ complex numbers $b_{n}, n=1,2, \ldots, k$

$$
\langle B\rangle=\operatorname{diag}\left(b_{1}, b_{2}, \ldots, b_{k}\right), \quad \sum_{n=1}^{k} b_{n}=0,
$$

where the condition on their sum ensures that the center of mass of the NS5-brane system does not change in accordance with the fact that the corresponding $U(1)$ degree of freedom is not part of the interacting LST. Since $\langle A\rangle=0$ only the operators with $l=0$ from (3.5) and (3.6) have non-vanishing VEVs. Hence, the holographic dictionary becomes

$$
\operatorname{tr}\left(B^{2 j+2}\right) \leftrightarrow e^{-\varphi-\bar{\varphi}} \psi^{+} \bar{\psi}^{+} \Phi_{j ; j, j}^{s u} e^{-q(j+1) \phi},
$$

since $\psi^{+}, \bar{\psi}^{+}$have $\left(J_{3}^{\text {tot }}, \bar{J}_{3}^{\text {tot }}\right)=(1,1)$.

A different representation of these operators comes from the decomposition

$$
\mathbb{R}_{\phi} \times S U(2)_{k} \equiv \mathbb{R}_{\phi} \times\left(U(1)_{k} \times \frac{S U(2)_{k}}{U(1)}\right) / \mathbb{Z}_{k}
$$

The infinite cylinder $\mathbb{R}_{\phi} \times U(1)_{k}$ is parameterized by $\phi$ and $Y$ with the latter defined as

$$
J_{3}^{\mathrm{tot}}=\frac{i}{q} \partial Y .
$$

The $\mathcal{N}=2$ minimal model $S U(2)_{k} / U(1)$ can be described in terms of a Landau-Ginzburg superfield $\chi$ with superpotential

$$
W=\chi^{k}
$$

Then we can write

$$
\psi^{+} \bar{\psi}^{+} \Phi_{j ; j, j}^{s u} e^{-q(j+1) \phi}=\chi^{k-2(j+1)} e^{-q(j+1) \Phi}
$$


where $\Phi$ is a chiral superfield whose bottom component is $\phi-i Y$ (and, as usual, we will denote both of them by the same symbol from now on). The perturbed Lagrangian can be written as

$$
\mathcal{L}=\mathcal{L}_{0}+\left(\sum_{j} \lambda_{j} \int d^{2} \theta \chi^{k-2(j+1)} e^{-q(j+1) \Phi}+\text { c.c. }\right)
$$

where the couplings $\lambda_{j}$ are specified in terms of the locations of the NS5-branes on the $B$ plane: $\lambda_{j}=\frac{1}{k}\left\langle\operatorname{tr}\left(B^{2 j+2}\right)\right\rangle$, with the proportionality factor $1 / k$ being included so that the coupling, for generic NS5-distributions, is appropriately normalized.

So far the discussion applies to a configuration of NS5-branes located at a point in $\mathbb{R}^{4}$, where the dual conformal field theory is (2.7), and (3.1) associates deformations of the NS5branes around the point with marginal operators in the CHS background. If, instead, we are interested in deformations of the circular configuration of NS5-branes, which admits an exact CFT description in terms of (2.11), we would like to associate VEVs of the chiral operators $\operatorname{tr}\left(\Phi^{i_{1}} \Phi^{i_{2}} \cdots \Phi^{i_{2 j+2}}\right)$ with marginal operators in (2.11). A way to find the latter is to consider (2.11) as a deformation of $\mathbb{R}_{\phi} \times S U(2)_{k} \simeq \mathbb{R}_{\phi} \times\left(U(1)_{k} \times \frac{S U(2)_{k}}{U(1)}\right) / \mathbb{Z}_{k}$ with the cylinder $\mathbb{R}_{\phi} \times U(1)_{k}$ being deformed to the $S L(2, \mathbb{R})_{k} / U(1)$ coset theory. For the operators of interest the dictionary becomes

$$
\operatorname{tr}\left(B^{2 j+2}\right) \leftrightarrow e^{-\varphi-\bar{\varphi}} V_{\frac{k}{2}-j-1 ;-\frac{k}{2}+j+1,-\frac{k}{2}+j+1}^{s u} V_{j ; j+1, j+1}^{s l}
$$

The corresponding worldsheet deformations are

$$
\mathcal{L}=\mathcal{L}_{0}+\left(\lambda_{j} G_{-\frac{1}{2}} \bar{G}_{-\frac{1}{2}} V_{\frac{k}{2}-j-1 ;-\frac{k}{2}+j+1,-\frac{k}{2}+j+1}^{s u} V_{j ; j+1, j+1}^{s l}+\text { c.c. }\right)
$$

and we can use (2.12) and (2.16) to check that they are indeed marginal.

A very interesting feature of the CFT operators in (3.8) and (3.14) is that they are (chiral, chiral) [30]. One can verify, using the formulas in App. A for (3.8) and in App. B and App. C for (3.14), that

$$
\begin{aligned}
& G^{+}(z) \psi^{+} \bar{\psi}^{+} \Phi_{j ; j, j}^{s u} e^{-q(j+1) \phi}(w, \bar{w}) \sim 0, \\
& G^{+}(z) V_{\frac{k}{2}-j-1 ;-\frac{k}{2}+j+1,-\frac{k}{2}+j+1}^{s u} V_{j ; j+1, j+1}^{s l}(w, \bar{w}) \sim 0
\end{aligned}
$$

and similarly for the antiholomorphic sector. Since the conformal weights of chiral operators are fixed in terms of their $R$-charges, the corresponding worldsheet deformations are exactly marginal. It is interesting to notice that spreading the NS5-branes on the $A$-plane while keeping them at a fixed point in the $B$-plane, $\langle B\rangle=0$, corresponds holographically to CFT operators that are (chiral, antichiral). Performing, instead, general (non-planar) deformations of the NS5-branes where both $\langle A\rangle$ and $\langle B\rangle$ are non-zero triggers non-chiral operators and therefore we lose exact marginality. Finally, we mention that interesting properties of the chiral ring comprised by the operators (3.14) has been studied in [31, 32]. 
We proceed now with the computation of the worldsheet deformation in (3.15). The supercurrent is $G=\frac{1}{\sqrt{2}}\left(G^{+}+G^{-}\right)$and the action of the supercharge $G_{-\frac{1}{2}}$ on the operator is captured by the simple pole of its OPE with $G$. As we mentioned above, the operators under consideration are chiral and hence the singularities in their OPE with $G$ will come only from the action of $G^{-}$. Using the decomposition of the supersymmetric coset primaries presented in the previous subsection, the decomposition of the supercurrents from App. B and the parafermionic OPEs from App. C, we can derive the following expressions

$$
\begin{aligned}
G^{-s u}(z) V_{\frac{k}{2}-j-1 ;-\frac{k}{2}+j+1,-\frac{k}{2}+j+1}^{s u}(w, \bar{w}) & \sim \frac{\sqrt{2} \alpha_{1}}{z-w} \psi_{\frac{k}{2}-j-1 ;-\frac{k}{2}+j+2,-\frac{k}{2}+j+1} \\
& \times \exp \left(i \frac{2(j+1)}{\sqrt{k(k-2)}} P_{\mathrm{L}}+i \frac{2(j+1)-k}{\sqrt{k(k-2)}} P_{\mathrm{R}}\right)
\end{aligned}
$$

and

$$
\begin{aligned}
G^{-s l}(z) V_{j ; j+1, j+1}^{s l}(w, \bar{w}) & \sim \frac{\sqrt{2} \alpha_{2}}{z-w} \pi_{j ; j+2, j+1} \\
& \times \exp \left(i \frac{2(j+1)-k}{\sqrt{k(k+2)}} Q_{\mathrm{L}}+i \frac{2(j+1)}{\sqrt{k(k+2)}} Q_{\mathrm{R}}\right),
\end{aligned}
$$

with coefficients

$$
\alpha_{1}=\frac{1}{\sqrt{k}}(k-2(j+1)), \quad \alpha_{2}=\frac{2(j+1)}{\sqrt{k}} .
$$

Similar expressions hold for the actions of the antiholomorphic supercurrents.

The operator we add to the worldsheet Lagrangian comes from the action of the total supercharges $G^{-}=G^{-s u}+G^{-s l}$ and $\bar{G}^{-}=\bar{G}^{-s u}+\bar{G}^{-s l}$ on

$$
V_{\frac{k}{2}-j-1 ;-\frac{k}{2}+j+1,-\frac{k}{2}+j+1}^{s u} V_{j ; j+1, j+1}^{s l}:=\mathcal{V}^{s u} \mathcal{V}^{s l},
$$

so that the result, after taking into account the factor $1 / 2$ coming from the definitions of $G$ and $\bar{G}$, schematically reads:

$$
\frac{1}{2}\left(\mathcal{V}^{s l}\left(G^{-s u} \bar{G}^{-s u} \mathcal{V}^{s u}\right)+\left(G^{-s u} \mathcal{V}^{s u}\right)\left(\bar{G}^{-s l} \mathcal{V}^{s l}\right)+\left(G^{-s l} \mathcal{V}^{s l}\right)\left(\bar{G}^{-s u} \mathcal{V}^{s u}\right)+\mathcal{V}^{s u}\left(G^{-s l} \bar{G}^{-s l} \mathcal{V}^{s l}\right)\right)
$$

The total deformation of the Lagrangian is given by (3.21) multiplied by the couplings $\lambda_{j}$ and upon adding to it its complex conjugate so that the total expression is real. Explicitly, the four operators in (3.21) read:

$$
\begin{aligned}
& C_{j, k}^{(1)} \alpha_{1}^{2} \psi_{\frac{k}{2}-j-1 ;-\frac{k}{2}+j+2,-\frac{k}{2}+j+2} \pi_{j ; j+1, j+1} e^{i 2(j+1) H}, \\
& C_{j, k}^{(2)} \alpha_{1} \alpha_{2} \psi_{\frac{k}{2}-j-1 ;-\frac{k}{2}+j+2,-\frac{k}{2}+j+1} \pi_{j ; j+1, j+2} e^{i 2(j+1) H_{\mathrm{L}}+i(2(j+1)-k) H_{\mathrm{R}}}, \\
& C_{j, k}^{(3)} \alpha_{2} \alpha_{1} \psi_{\frac{k}{2}-j-1 ;-\frac{k}{2}+j+1,-\frac{k}{2}+j+2} \pi_{j ; j+2, j+1} e^{i(2(j+1)-k) H_{\mathrm{L}}+i 2(j+1) H_{\mathrm{R}}}, \\
& C_{j, k}^{(4)} \alpha_{2}^{2} \psi_{\frac{k}{2}-j-1 ;-\frac{k}{2}+j+1,-\frac{k}{2}+j+1} \pi_{j ; j+2, j+2} e^{i(2(j+1)-k) H},
\end{aligned}
$$


where $H$ is a free boson defined as

$$
H=\frac{1}{\sqrt{k}}\left(\frac{P}{\sqrt{k-2}}+\frac{Q}{\sqrt{k+2}}\right) .
$$

The $C_{j, k}^{(a)}$ 's are numbers that depend on the relative cocycles that we should include in principle when bosonizing the fermions of the supersymmetric cosets. Their explicit form is not necessary for our purposes, as we will be interested in the large- $k$ limit in which we can infer easily that these numbers for $a=1,2,4$ can be taken to be 1 and for $a=3$ to be -1 . This is essentially due to the fact that in the third term in (3.21) the order of the exponentials corresponding to bosonized fermions for the compact and the non-compact cosets has been interchanged compared to the third and fourth terms, whereas in (3.24) the order has been restored.

The four operators above are partners under $\mathcal{N}=1$ worldsheet supersymmetry since the deformations under consideration should leave space-time supersymmetry (and hence worldsheet supersymmetry as well) unbroken. In the semiclassical limit $k \rightarrow \infty$ and for $j<<k$, where we can think of them as deformations of the supersymmetric $\sigma$-model Lagrangian with metric (2.29), the first one, Eq. (3.22), would be a purely bosonic deformation. Indeed, the contribution of the fermions, captured by the exponential involving the bosonized field $H$, would be vanishing. Accordingly, the operators (3.23) and (3.24) correspond to 2-fermion terms while (3.25) is a 4 -fermion interaction term. In the next section we will identify explicitly the semiclassical limit of these operators with the corresponding $\sigma$-model deformations. An exception to this picture would be the case of $j=(k-2) / 2$ which is of order $k$. This will be treated separately and we will show that it corresponds also to a nice geometrically interpreted deformation.

\subsection{Holographic dictionary: applications}

It is time now to employ the holographic dictionary presented above in order to uncover how changing the configuration of the NS5-branes affects the underlying conformal field theory description.

\subsubsection{Point to circle}

We start with a warm-up example taken from [8]. Let us distribute the NS5-branes symmetrically on a circle of radius $r_{0}$ on the $B$-plane so that the eigenvalues $b_{n}$ take the form

$$
b_{n}=r_{0} e^{\frac{2 \pi i}{k} n} \text {. }
$$

Since $\operatorname{tr}\left\langle B^{l}\right\rangle=0$ for $l<k$ we have

$$
\lambda_{j}=\mu \delta_{j, \frac{k-2}{2}},
$$

with $\mu \sim r_{0}^{k}$. The value of the spin $j=(k-2) / 2$ is very special since the multi-trace contributions, mentioned in footnote 3 , are vanishing. The deformed worldsheet theory is

$$
\mathcal{L}=\mathcal{L}_{0}+\left(\mu \int d^{2} \theta e^{-\frac{1}{q} \Phi}+\text { c.c. }\right)
$$


which we recognize as the $\mathcal{N}=2$ Liouville theory. Combined with the $\mathcal{N}=2$ minimal model and upon orbifolding with $\mathbb{Z}_{k}$, the theory thus obtained is equivalent to (2.11), which indeed describes the near-horizon geometry of a circular configuration of NS5-branes [6].

\subsubsection{Circle to circle}

It is interesting to consider the operator with $j=\frac{k-2}{2}$, but in the non-singular background (2.11). This operator should correspond to changes of the radius of the circular distribution of the NS5-branes. If we change the radius from $r_{0}$ to $r_{0}+\delta$, we can either add to the original CHS theory the $j=\frac{k-2}{2}$ operator with coefficients $\mu \sim r_{0}^{k}$ and $\mu \sim\left(r_{0}+\delta\right)^{k}$ respectively or, equivalently, deform the $S L(2, \mathbb{R}) / U(1) \times S U(2) / U(1)$ with the corresponding operator bearing a coefficient $\left(r_{0}+\delta\right)^{k}-r_{0}^{k} \simeq k r_{0}^{k-1} \delta$.

The operator we use in this case in (3.15) reads:

$$
V_{0 ; 0,0}^{s u} V_{\frac{k}{2}-1 ; \frac{k}{2}, \frac{k}{2}}^{s l}
$$

Note that in this case the spin $j$ is of the same order of magnitude as $k$, but as long as we stay within the exact framework this does not present a problem. The correspondence with the semiclassical expressions for the primaries will be done soon using the equivalence relation $(2.19)$.

Since for this value of $j$ the coefficients $\alpha_{1}$ vanishes, the worldsheet Lagrangian changes by

$$
\alpha_{2}^{2} \psi_{0 ; 0,0} \pi_{\frac{k}{2}-1 ; \frac{k}{2}+1, \frac{k}{2}+1}+\text { c.c. }=\alpha_{2}^{2} \pi_{\frac{k}{2}-1 ; \frac{k}{2}+1, \frac{k}{2}+1}+\text { c.c. },
$$

since $\psi_{0 ; 0,0}$ is the identity field. It can be verified using (2.18) that this operator is marginal. Notice also that this is a purely bosonic deformation, since the bosonic field $H$ relating to the fermions doesn't appear. This is an explicit example which indicates, among other things, that the rôle of the various terms in (3.22)-(3.25) for low spin when $j \ll k$, as explained before the beginning of this subsection, could be completely different for high spins having $j \sim \frac{k}{2}$.

We would like now to find a semiclassical expression for the non-compact parafermionic primary $\pi_{\frac{k}{2}-1 ; \frac{k}{2}+1, \frac{k}{2}+1}$. Notice that (B.17) yields

$$
\pi(z) \pi_{\frac{k}{2}-1 ; \frac{k}{2}, \frac{k}{2}}(w, \bar{w}) \sim \frac{1}{(z-w)^{\frac{2}{k+2}}} \pi_{\frac{k}{2}-1 ; \frac{k}{2}+1, \frac{k}{2}+1}(w, \bar{w}),
$$

and similarly with $\pi(z)$ replaced by $\bar{\pi}(\bar{z})$. In the semiclassical limit $k \rightarrow \infty$ we can think of $\pi_{\frac{k}{2}-1 ; \frac{k}{2}+1, \frac{k}{2}+1}$ as a composite field $\pi \bar{\pi} \pi_{\frac{k}{2}-1 ; \frac{k}{2}, \frac{k}{2}}$ and their conformal dimensions match only to leading order in $1 / k$, as it should be. Now, the equivalence between non-compact parafermionic primaries can be used to replace $\pi_{\frac{k}{2}-1 ; \frac{k}{2}, \frac{k}{2}}$ by $\pi_{0 ;-1,-1}$. This is necessary as the semiclassical limit of the expression for the primary $\pi_{\frac{k}{2}-1 ; \frac{k}{2}, \frac{k}{2}}$ does not seem to be welldefined since its spin is of order $k$. The punch-line is that for large $k$ the leading deformation 
of the worldsheet Lagrangian is through the operator

$$
\alpha_{2}^{2} \pi \bar{\pi} \pi_{0 ;-1,-1}+\text { c.c. } \sim \alpha_{2}^{2}\left(\frac{\partial \rho \bar{\partial} \rho}{\cosh ^{2} \rho}-\frac{\partial \omega \bar{\partial} \omega}{\sinh ^{2} \rho}\right),
$$

where we have used the expressions $(2.27),(2.28)$ and $(2.32)$ for the semiclassical $S L(2, \mathbb{R}) / U(1)$ parafermions and primaries.

The above operator is a deformation of the $\sigma$ model (2.29) and more precisely only of its $S L(2, \mathbb{R}) / U(1)$ part. The $\sigma$ model of the latter is defined in (2.26) and appending (3.33) to it with coefficient $\frac{1}{k} \alpha_{2}^{2} k r_{0}^{k-1} \delta \sim \epsilon k$ changes the above data to

$$
\frac{d s^{2}}{k}=\left(1+\frac{\epsilon}{\cosh ^{2} \rho}\right) d \rho^{2}+\left(\operatorname{coth}^{2} \rho-\frac{\epsilon}{\sinh ^{2} \rho}\right) d \omega^{2}, \quad e^{-2 \Phi}=\sinh ^{2} \rho .
$$

Now, a coordinate redefinition $\rho \rightarrow \rho-\frac{\epsilon}{2} \tanh \rho$ brings the deformed metric back to its original form but changes the profile of the dilaton as

$$
e^{-2 \Phi}=(1-\epsilon) \sinh ^{2} \rho
$$

In the T-dual theory, i.e. the cigar, the dilaton takes the form

$$
e^{-2 \Phi}=(1-\epsilon) \cosh ^{2} \rho
$$

and its value at $\rho=0$, i.e. at the tip of the cigar, is related to the radius of the circle on which we put the NS5-branes [33]. Here we see explicitly that changing this radius has the expected effect on the value of the dilaton at the tip.

\subsubsection{Circle to ellipsis}

Let us now consider a deformation of the circular configuration of NS5-branes to an ellipsis. If the latter has radii $r_{1}=r_{0}(1+\epsilon)$ and $r_{2}=r_{0}(1-\epsilon)$ the positions of the NS5-branes on the $x^{8}-x^{9}$ plane are parameterized by $\left\langle\Phi_{n}^{8}\right\rangle=r_{1} \cos \left(\phi_{n}\right),\left\langle\Phi_{n}^{9}\right\rangle=r_{2} \sin \left(\phi_{n}\right)$ with $\phi_{n}=\frac{2 \pi}{k} n, n=$ $1, \ldots, k$. Since $\langle B\rangle=\left\langle\Phi^{8}\right\rangle+i\left\langle\Phi^{9}\right\rangle$ we have

$$
b_{n}=r_{0}\left(e^{\frac{2 \pi i}{k} n}+\epsilon e^{-\frac{2 \pi i}{k} n}\right) .
$$

We can think of $\epsilon$ as controlling the deformation of a circle of radius $r_{0}$ to an ellipsis. The couplings $\lambda_{j}$ are proportional to

$$
\left\langle\operatorname{tr}(B)^{m}\right\rangle=k r^{m} \sum_{l=0}^{m}\left(\begin{array}{c}
m \\
l
\end{array}\right) \epsilon^{l}\left(\delta_{2 l-m, k}+\delta_{2 l, m}+\delta_{2 l-m,-k}\right),
$$

where $m=2 j+2=2,3, \ldots, k$. Operators with a given $j \in \mathbb{N}$ result in corrections of order $\epsilon^{j+1}$. The leading deformation, which is of order $\epsilon$, appears for $m=2$ and therefore $j=0$. The corresponding operator we use in (3.15) is

$$
V_{\frac{k}{2}-1 ;-\frac{k}{2}+1,-\frac{k}{2}+1}^{s u} V_{0 ; 1,1}^{s l}
$$


and we can read from (3.22)-(3.25) the associated Lagrangian deformations:

$$
\begin{aligned}
& C_{0, k}^{(1)} \alpha_{1}^{2} \psi_{\frac{k}{2}-1 ;-\frac{k}{2}+2,-\frac{k}{2}+2} \pi_{0 ; 1,1} e^{2 i H}, \\
& C_{0, k}^{(2)} \alpha_{1} \alpha_{2} \psi_{\frac{k}{2}-1 ;-\frac{k}{2}+2,-\frac{k}{2}+1} \pi_{0 ; 1,2} e^{2 i H_{\mathrm{L}}+i(2-k) H_{\mathrm{R}}}, \\
& C_{0, k}^{(3)} \alpha_{2} \alpha_{1} \psi_{\frac{k}{2}-1 ;-\frac{k}{2}+1,-\frac{k}{2}+2} \pi_{0 ; 2,1} e^{i(2-k) H_{\mathrm{L}}+2 i H_{\mathrm{R}}}, \\
& C_{0, k}^{(4)} \alpha_{2}^{2} \psi_{\frac{k}{2}-1 ;-\frac{k}{2}+1,-\frac{k}{2}+1} \pi_{0 ; 2,2} e^{i(2-k) H} .
\end{aligned}
$$

Recall also that we have to add to the operators above their complex conjugates.

We would like now to understand the semiclassical limit of these operators. We begin with the exponentials and in the large- $k$ limit we have the following correspondences

$$
\begin{array}{rlrl}
e^{2 i H} & \rightarrow 1, & h=\bar{h} \simeq 0, \\
e^{2 i H_{\mathrm{L}}+i(2-k) H_{\mathrm{R}}} \rightarrow e^{-i\left(P_{\mathrm{R}}+Q_{\mathrm{R}}\right)}, & h \simeq 0, \quad \bar{h} \simeq 1-\frac{4}{k}, \\
e^{i(2-k) H_{\mathrm{L}}+i 2 H_{\mathrm{R}}} \rightarrow e^{-i\left(P_{\mathrm{L}}+Q_{\mathrm{L}}\right)}, & h \simeq 1-\frac{4}{k}, \quad \bar{h} \simeq 0, \\
e^{i(2-k) H} \rightarrow e^{-i(P+Q)}, & h=\bar{h} \simeq 1-\frac{4}{k},
\end{array}
$$

where we have indicated the semiclassical expressions explicitly in terms of the original bosons $P$ and $Q$, along with the conformal dimension up to order $\mathcal{O}(1 / k)$ for which we are interested in.

For the non-compact parafermionic primaries the semiclassical expressions can be read from (2.32) and (2.34). For the compact parafermionic primaries similar formulas would be illdefined semiclassicaly (an analogous situation was encountered for the non-compact primaries in the previous example) and we should find them indirectly. For instance the dimension of $\psi_{\frac{k}{2}-1 ;-\frac{k}{2}+1,-\frac{k}{2}+1}$ is 0 and indeed the parafermionic equivalence (2.15) relates this operator to the identity $\psi_{0 ; 0,0}$. Furthermore, for $\psi_{\frac{k}{2}-1 ;-\frac{k}{2}+2,-\frac{k}{2}+1}$ the dimension of the left part is $h=1-1 /(k-2)$, i.e. the same as the one for the compact parafermions and moreover, using the OPE (B.10) (for $j=k / 2-1, m=-k / 2+1$ and remembering first to shift in that expression $k \rightarrow k-2$ ) we conclude that it can be identified with that. Hence, we have the exact identifications

$$
\psi_{\frac{k}{2}-1 ;-\frac{k}{2}+2,-\frac{k}{2}+2}=\psi \bar{\psi}, \quad \psi_{\frac{k}{2}-1 ;-\frac{k}{2}+2,-\frac{k}{2}+1}=\psi, \quad \psi_{\frac{k}{2}-1 ;-\frac{k}{2}+1,-\frac{k}{2}+2}=\bar{\psi} .
$$

We can now assemble everything using the expressions (2.22) and (2.23) for the parafermion fields and (2.32) and (2.34) for the non-compact primaries. The deforming operators in the semiclassical limit read:

$$
\begin{aligned}
& k \frac{1}{g_{--}^{2}} \psi \bar{\psi}, \\
& -\frac{2 i}{g_{--}^{3}}\left(g_{-+} \psi e^{-i\left(P_{\mathrm{R}}+Q_{\mathrm{R}}\right)}+g_{+-} \bar{\psi} e^{-i\left(P_{\mathrm{L}}+Q_{\mathrm{L}}\right)}\right), \\
& \frac{2}{k} \frac{2 g_{+-} g_{-+}-1}{g_{--}^{4}} e^{-i(P+Q)},
\end{aligned}
$$


where, compared with (3.41), we have replaced the constant factors $a_{1,2}$ by their leading $1 / k$ behavior and in addition we have set the co-cycle dependent factors $C_{0, k}^{(a)}$ to their semiclassical values as described below (3.26). It is understood that $\psi, \bar{\psi}$ and the various group elements are represented in terms of the target-space variables as in Sec. 2.1. In the next section we will identify them with the $\mathcal{N}=1 \sigma$ model deformations induced by changing the circular distribution of NS5-branes to an elliptical one. In particular, the first line above will be related to the deformation of the bosonic part, thus recovering the result in [17], the second line will correspond to the deformation of the fermion bilinears and the third line to the deformation of the quartic, in the fermions, term.

\subsubsection{General circle deformations}

We consider now general deformations of a circular distribution of NS5-branes. Their positions in the $B$-plane are encoded by

$$
b_{n}=r_{0}\left(e^{\frac{2 \pi i n}{k}}+\epsilon_{n}\right), \quad n=1, \ldots, k, \quad \sum_{n=0}^{k} \epsilon_{n}=0
$$

Here $\epsilon_{n}<<1$ are complex numbers indicating the shift of the position of the $n$-th NS5-brane relative to the original circle. To leading order in $\epsilon_{n}$ all operators in (3.14) for which the sum

$$
\sum_{n=0}^{k} \epsilon_{n} e^{\frac{2 \pi i n(2 j+1)}{k}}
$$

is non-zero, contribute.

It is natural to proceed by looking for a deformation of the circle distribution so that only a single operator is turned on. In other words, we would like a set of numbers $\epsilon_{n}$ with the property that their sum is zero and all sums of the form (3.45) are zero except for one given

value of $j$. Obviously such a set exists and it is given by $\epsilon_{n}=\epsilon e^{-\frac{2 \pi i n(2 j+1)}{k}}$. For instance, for $j=0$ we retrieve the deformation to the ellipsis studied previously, while for $j=(k-2) / 2$ we see that, as expected, we change the radius $r_{0} \rightarrow r_{0}(1+\epsilon)$. The perturbed positions of the NS5 branes are

$$
b_{n}=r_{0}\left(e^{\frac{2 \pi i n}{k}}+\epsilon e^{-\frac{2 \pi i n(2 j+1)}{k}}\right), \quad n=1, \ldots, k .
$$

An important feature of the distribution (3.46) is that the NS5-branes are not uniformly distributed. In order to understand that, we should consider the continuum limit of (3.46), which will be also important for the considerations of the next section. In this limit $k \rightarrow \infty$ and the discrete angles $\phi_{n}=\frac{2 \pi n}{k}$ are replaced by a continuous angle $\phi \in[0,2 \pi]$. The discrete distribution (3.46) approaches

$$
b(\phi)=r_{0} e^{i \phi}\left(1+\epsilon e^{-2 i(j+1) \phi}\right)
$$


and to leading order we can think of that as deforming the radius $r_{0}$ of the original circular distribution as $r_{0} \rightarrow r_{0}(1+\epsilon \cos 2(j+1) \psi)$ and also changing the angle as $\phi=\psi+\epsilon \sin 2(j+$ 1) $\psi$. Hence the original uniform angular distribution with measure $d \psi$ is replaced by

$$
d \psi \lambda(\psi), \quad \lambda(\psi)=1+2 \epsilon(j+1) \cos 2(j+1) \psi
$$

We would like now to understand the semiclassical limit of the general operators (3.22)(3.25). As in the case of the elliptical deformation we begin with the exponentials and in the large- $k$ limit we have the following correspondences

$$
\begin{array}{rlrl}
e^{2 i(j+1) H} & \rightarrow 1, & h=\bar{h} \simeq 0, \\
e^{2 i(j+1) H_{\mathrm{L}}+i[2(j+1)-k] H_{\mathrm{R}}} \rightarrow e^{-i\left(P_{\mathrm{R}}+Q_{\mathrm{R}}\right)}, & h \simeq 0, \quad \bar{h} \simeq 1-\frac{4(j+1)}{k}, \\
e^{i[2(j+1)-k] H_{\mathrm{L}}+2 i(j+1) H_{\mathrm{R}}} \rightarrow e^{-i\left(P_{\mathrm{L}}+Q_{\mathrm{L}}\right)}, & h \simeq 1-\frac{4(j+1)}{k}, \quad \bar{h} \simeq 0, \\
e^{i[2(j+1)-k] H} \rightarrow e^{-i(P+Q)}, & h=\bar{h} \simeq 1-\frac{4(j+1)}{k},
\end{array}
$$

where we have indicated the semiclassical expressions explicitly in terms of the original bosons $P$ and $Q$, along with the conformal dimension up to order $\mathcal{O}(1 / k)$ for which we are interested in.

Considering the compact primaries, as in the previous example of the elliptical deformation, we will find them indirectly, but extra care is needed as some of them arise as composite operators. The parafermionic equivalence (2.15) implies that $\psi_{\frac{k}{2}-j-1 ;-\frac{k}{2}+j+1,-\frac{k}{2}+j+1}$ is related to the operator $\psi_{j ; j, j}$. Furthermore, the primary $\psi_{\frac{k}{2}-j-1 ;-\frac{k}{2}+j+2,-\frac{k}{2}+j+1}$ arises as a composite operator of the compact parafermion $\psi$ with $\psi_{j ; j, j}$. This can be seen by using the OPE

$$
\psi(z) \psi_{\frac{k}{2}-j-1 ;-\frac{k}{2}+j+1,-\frac{k}{2}+j+1}(w, \bar{w}) \sim \frac{k-2(j+1)}{\sqrt{k-2}}(z-w)^{\frac{-2 j}{k-2}} \psi_{\frac{k}{2}-j-1 ;-\frac{k}{2}+j+2,-\frac{k}{2}+j+1}(w, \bar{w}),
$$

arising from (B.10) (remembering to shift $k \rightarrow k-2$ ) and the parafermionic equivalence. Hence, we have the exact identifications

$$
\begin{array}{lll}
\psi_{\frac{k}{2}-j-1 ;-\frac{k}{2}+j+2,-\frac{k}{2}+j+2}=\psi_{j ; j, j} \psi \bar{\psi}, & h=\bar{h} \simeq 1-\frac{j+1}{k}, \\
\psi_{\frac{k}{2}-j-1 ;-\frac{k}{2}+j+2,-\frac{k}{2}+j+1}=\psi_{j ; j, j} \psi, & h \simeq 1-\frac{j+1}{k}, \bar{h}=\frac{j}{k}, \\
\psi_{\frac{k}{2}-j-1 ;-\frac{k}{2}+j+1,-\frac{k}{2}+j+2}=\psi_{j ; j, j} \bar{\psi}, & h=\frac{j}{k}, \bar{h} \simeq 1-\frac{j+1}{k},
\end{array}
$$

where we have indicated the conformal dimensions of the different terms. We emphasize that, in the product of two operators of the compact coset the dimension is not just the sum of the dimensions. One actually should think of them as one composite operator defined through the OPE (B.10). The above identifications are correct as merely a simple product, when we will later perform the comparison with the $\mathcal{N}=1 \sigma$ model. 
We can now assemble everything using the expressions (2.22) and (2.23) for the parafermion fields and (2.32) and (2.34) for the non-compact primaries. The deforming operators in the semiclassical limit read:

$$
\begin{aligned}
& k \frac{\tilde{g}_{+-}^{2 j}}{g_{--}^{2(j+1)}} \psi \bar{\psi} \\
& -2(j+1) \frac{i \tilde{g}_{+-}^{2 j}}{g_{--}^{2 j+3}}\left(g_{-+} \psi e^{-i\left(P_{\mathrm{R}}+Q_{\mathrm{R}}\right)}+g_{+-} \bar{\psi} e^{-i\left(P_{\mathrm{L}}+Q_{\mathrm{L}}\right)}\right), \\
& \frac{2(j+1)}{k} \tilde{g}_{+-}^{2 j} \frac{2(j+1) g_{+-} g_{-+}-1}{g_{--}^{2(j+2)}} e^{-i(P+Q)},
\end{aligned}
$$

to which we should add their complex conjugates.

\section{Deformations of NS5-branes: $\sigma$ model approach and comparison}

In this section we perform a complete comparison between a general infinitesimal deformation of a circular distribution of NS5-branes on the same plane, as it results from two different approaches. Namely, either from the corresponding $\mathcal{N}=1$ supersymmetric $\sigma$ model viewpoint or from the exact CFT approach of the previous section as an exactly marginal perturbation based on the holographic conjecture. First we consider the simpler case of the circular distribution deformed into a small ellipsis and complete the analysis of [17] which was performed only for the bosonic part of the supersymmetric action. Subsequently, we consider the general planar deformation. The two approaches are in complete agreement in their semiclassical range of common validity, including numerical coefficients.

\subsection{Neveu-Schwarz five-branes on an ellipsis}

Let us consider a system of $k$ NS5-branes distributed on the circumference of an ellipsis with axes $\ell_{1}$ and $\ell_{2}$ according to the density

$$
\rho(\mathbf{x})=\frac{1}{\pi \ell_{1} \ell_{2}} \delta\left(1-\frac{\left(x^{8}\right)^{2}}{\ell_{1}^{2}}-\frac{\left(x^{9}\right)^{2}}{\ell_{2}^{2}}\right) \delta\left(x^{6}\right) \delta\left(x^{7}\right) .
$$

The supergravity solution corresponding to (4.1) along with its T-dual was constructed and studied in detail in [17]. Here we are interested in the infinitesimal deformation of a circular distribution with $\ell_{1}=\ell_{2}$, which admits the exact CFT description (2.11), to an ellipsis with $\ell_{1}=r_{0}(1+\epsilon), \ell_{2}=r_{0}(1-\epsilon)$. Given a density distribution one can construct the corresponding supergravity solution from (2.1)-(2.3). In our case it is convenient to parameterize the Cartesian coordinates in a way appropriate to the density (4.1) as [17]

$$
\mathbf{x}=\ell_{1}\left(\sinh \rho \cos \theta \cos \tau, \sinh \rho \cos \theta \sin \tau, \cosh \rho \sin \theta \cos \psi, \sqrt{\sinh ^{2} \rho+\frac{\ell_{2}^{2}}{\ell_{1}^{2}}} \sin \theta \sin \psi\right)
$$


In the limit $\ell_{1}=\ell_{2}=r_{0}$ one obtains the supergravity solution representing a uniform continuous NS5-brane distribution [6] which semiclassicaly is described, after a proper Tduality, by the $\sigma$ model (2.29).

The leading order correction to (2.29), corresponding to an infinitesimal deformation of the circle towards an ellipsis and after the proper T-duality and a reparameterization, reads [17] (see Eq. (38) in that Ref.):

$$
d s^{(1) 2}=\frac{2 \epsilon k}{\cosh ^{2} \rho}\left[\cos 2(\omega-\varphi)\left(d \theta^{2}-\tan ^{2} \theta d \varphi^{2}\right)+2 \sin 2(\omega-\varphi) \tan \theta d \varphi d \theta\right]
$$

where we note the relation of the angular variables as compared to (4.2) is $\varphi=\tau$ and $\omega=\tau+\psi$. The full metric is the sum of the metric in (2.29) and of that in (4.3), whereas the antisymmetric tensor remains vanishing. It will be convenient to work with a coframe $\left\{e^{\hat{\imath}}\right\}$ such that the full metric takes the form

$$
d s^{2}=\eta_{\hat{\imath} \hat{\jmath}} e^{\hat{\imath}} e^{\hat{\jmath}}
$$

with the hatted indices taking values $\hat{\imath}=\hat{1}, \ldots, \hat{4}$ and where

$$
\eta_{\hat{\imath} \jmath}=\left(\begin{array}{cccc}
0 & \frac{1}{2} & 0 & 0 \\
\frac{1}{2} & 0 & 0 & 0 \\
0 & 0 & 0 & \frac{1}{2} \\
0 & 0 & \frac{1}{2} & 0
\end{array}\right) .
$$

The relevant coframe, associated spin connections and curvature tensors can be retrieved from App. D by setting $n=2$ as it can be easily seen by comparing (4.3) with (D.1).

The $\mathcal{N}=1 \sigma$ model using tangent-space objects is $[34,35,36,37]$

$$
\begin{aligned}
S=\int d^{2} \sigma & \left(\eta_{\hat{\imath} \hat{\jmath}} e_{-}^{\hat{\imath}} e_{+}^{\hat{\jmath}}+i \eta_{\hat{\imath} \hat{\jmath}} \Psi_{+}^{\hat{\imath}}\left(\partial_{-} \Psi_{+}^{\hat{\jmath}}+\omega_{-\hat{k}}^{\hat{\jmath}} \Psi_{+}^{\hat{k}}\right)\right. \\
& \left.+i \eta_{\hat{\imath} \hat{\jmath}} \Psi_{-}^{\hat{\imath}}\left(\partial_{+} \Psi_{-}^{\hat{\jmath}}+\omega_{+\hat{k}}^{\hat{\jmath}} \Psi_{-}^{\hat{k}}\right)+\frac{1}{2} R_{\hat{\imath} \hat{\jmath} \hat{k}} \Psi_{+}^{\hat{\imath}} \Psi_{+}^{\hat{\jmath}} \Psi_{-}^{\hat{k}} \Psi_{-}^{\hat{\imath}}\right)
\end{aligned}
$$

where $e_{ \pm}^{\hat{\imath}}$ are the "chiral coframes", i.e. the coframes with their exterior differentials replaced by the appropriate chiral derivative, and similarly $\omega_{ \pm \hat{k}}^{\hat{j}}$ is the corresponding connection $\omega_{ \pm \hat{k}}^{\hat{\jmath}}=\omega_{\hat{\imath} \hat{k}}^{\hat{\jmath}} e_{ \pm}^{\hat{\imath}}$. Notice that the connection is the usual Levi-Civita connection since we have vanishing NS-NS three-form flux.

The fermions in (4.6) are coupled to the scalar sector of the theory. Hence, we would like to perform a field redefinition that renders them free, in the undeformed background, as in the CFT formulation in [8]. Therefore, we write the $\mathcal{N}=1 \sigma$ model (4.6) using the unperturbed 
(i.e. $\epsilon=0)$ connections from (D.6) and curvature tensors from (D.7) as

$$
\begin{aligned}
S_{0}=\int d^{2} \sigma & \left(\left(k\left(\partial_{+} \theta \partial_{-} \theta+\tan ^{2} \theta \partial_{+} \varphi \partial_{-} \varphi\right)+\frac{i}{2}\left(\Psi_{+}^{\hat{3}} \partial_{-} \Psi_{+}^{\hat{4}}+\Psi_{+}^{\hat{4}} \partial_{-} \Psi_{+}^{\hat{3}}\right)+i \Psi_{+}^{\hat{3}} \Psi_{+}^{\hat{4}} \omega_{-\hat{4}}^{\hat{4}}\right.\right. \\
& \left.+\frac{i}{2}\left(\Psi_{-}^{\hat{3}} \partial_{+} \Psi_{-}^{\hat{4}}+\Psi_{+}^{\hat{4}} \partial_{-} \Psi_{+}^{\hat{3}}\right)+i \Psi_{-}^{\hat{3}} \Psi_{-}^{\hat{4}} \omega_{+}^{\hat{4}}+2 R_{\hat{3} \hat{4} \hat{3} \hat{4}} \Psi_{-}^{\hat{3}} \Psi_{-}^{\hat{4}} \Psi_{+}^{\hat{3}} \Psi_{+}^{\hat{4}}\right) \\
& +\left(k\left(\partial_{+} \rho \partial_{-} \rho+\operatorname{coth}^{2} \rho \partial_{+} \omega \partial_{-} \omega\right)+\frac{i}{2}\left(\Psi_{+}^{\hat{1}} \partial_{-} \Psi_{+}^{\hat{2}}+\Psi_{+}^{\hat{2}} \partial_{-} \Psi_{+}^{\hat{1}}\right)+i \Psi_{+}^{\hat{1}} \Psi_{+}^{\hat{2}} \omega_{-\hat{2}}^{\hat{2}}\right. \\
& \left.\left.+\frac{i}{2}\left(\Psi_{-}^{\hat{1}} \partial_{+} \Psi_{-}^{\hat{2}}+\Psi_{+}^{\hat{2}} \partial_{-} \Psi_{+}^{\hat{1}}\right)+i \Psi_{-}^{\hat{1}} \Psi_{-}^{\hat{2}} \omega_{+\hat{2}}^{\hat{2}}+2 R_{\hat{1} \hat{2} \hat{\imath} \hat{2}} \Psi_{-}^{\hat{1}} \Psi_{-}^{\hat{2}} \Psi_{+}^{\hat{1}} \Psi_{+}^{\hat{2}}\right)\right) .
\end{aligned}
$$

The fermions $\Psi_{ \pm}^{\hat{1}}$ and $\Psi_{ \pm}^{\hat{2}}=\left(\Psi_{ \pm}^{\hat{1}}\right)^{*}$ belong to the non-compact coset while $\Psi_{ \pm}^{\hat{3}}$ and $\Psi_{ \pm}^{\hat{4}}=$ $\left(\Psi_{ \pm}^{\hat{3}}\right)^{*}$ belong to the compact one.

Now, the field redefinitions

$$
\partial_{ \pm} \varphi \rightarrow \partial_{ \pm} \varphi+\frac{1}{k} \Psi_{ \pm}^{3} \Psi_{ \pm}^{4}, \quad \partial_{ \pm} \omega \rightarrow \partial_{ \pm} \omega-\frac{1}{k} \Psi_{ \pm}^{1} \Psi_{ \pm}^{2}
$$

when substituted into (4.7), lead classically to the following $\sigma$ model

$$
\begin{aligned}
S=\int d^{2} \sigma & \left(k\left(\partial_{+} \theta \partial_{-} \theta+\tan ^{2} \theta \partial_{+} \phi \partial_{-} \phi\right)+\frac{i}{2}\left(\Psi_{+}^{\hat{3}} \partial_{-} \Psi_{+}^{\hat{4}}+\Psi_{+}^{\hat{4}} \partial_{-} \Psi_{+}^{\hat{3}}\right)\right. \\
& +\frac{i}{2}\left(\Psi_{-}^{\hat{3}} \partial_{+} \Psi_{-}^{\hat{4}}+\Psi_{-}^{\hat{4}} \partial_{+} \Psi_{-}^{\hat{3}}\right)+\frac{1}{k} \Psi_{-}^{\hat{3}} \Psi_{-}^{\hat{4}} \Psi_{+}^{\hat{3}} \Psi_{+}^{\hat{4}} \\
& +k\left(\partial_{+} \rho \partial_{-} \rho+\operatorname{coth}^{2} \rho \partial_{+} \omega \partial_{-} \omega\right)+\frac{i}{2}\left(\Psi_{+}^{\hat{1}} \partial_{-} \Psi_{+}^{\hat{2}}+\Psi_{+}^{\hat{2}} \partial_{-} \Psi_{+}^{\hat{2}}\right) \\
& \left.+\frac{i}{2}\left(\Psi_{-}^{\hat{1}} \partial_{+} \Psi_{-}^{\hat{2}}+\Psi_{-}^{\hat{2}} \partial_{+} \Psi_{-}^{\hat{1}}\right)-\frac{1}{k} \Psi_{-}^{\hat{1}} \Psi_{-}^{\hat{2}} \Psi_{+}^{\hat{1}} \Psi_{+}^{\hat{2}}\right) .
\end{aligned}
$$

The fermions have been decoupled from the scalars and are subject only to a Thirring-type interaction $[38,39]$. Therefore, they can be described through a compact boson of radius $R$ given by

$$
R-\frac{1}{R}+2 h=0,
$$

where $h$ is the coupling constant of the 4 -fermi interaction term. In our case the free boson radii for the two cosets turn out to be

$$
\begin{aligned}
& R_{S U(2) / U(1)}=-\frac{1}{k}+\sqrt{1+\frac{1}{k^{2}}} \simeq 1-\frac{1}{k} \simeq \sqrt{\frac{k-2}{k}}=R_{S U(2) / U(1)}^{\mathrm{CFT}}, \\
& R_{S L(2, \mathbb{R}) / U(1)}=\frac{1}{k}+\sqrt{1+\frac{1}{k^{2}}} \simeq 1+\frac{1}{k} \simeq \sqrt{\frac{k+2}{2}}=R_{S L(2, \mathbb{R}) / U(1)}^{\mathrm{CFT}} .
\end{aligned}
$$

The CFT radii at the right-hand side are those of the bosons $P$ and $Q$ that bosonize the free fermions of each supersymmetric coset and are consistent with the way these bosons enter into the exponentials in (C.1) and (C.3). The match here is only to leading order in $1 / k$ since the $\sigma$ model captures only the semiclassical features of the exact CFT and 
furthermore we have applied the field redefinitions classically ignoring any Jacobians from the transformations. These fermions correspond to the bosonized ones of the CFT side in terms of the fields $P$ and $Q$ defined in Sec. 2.2 as follows

$$
\begin{aligned}
& e^{i \phi_{2}} e^{-i Q_{\mathrm{L}}}=\Psi_{+}^{\hat{1}}, \quad e^{-i \phi_{2}} e^{i Q_{\mathrm{L}}}=\Psi_{+}^{\hat{2}}, \quad e^{i \phi_{1}} e^{-i P_{\mathrm{L}}}=\Psi_{+}^{\hat{3}}, \quad e^{-i \phi_{1}} e^{i P_{\mathrm{L}}}=\Psi_{+}^{\hat{4}}, \\
& e^{-i \phi_{2}} e^{-i Q_{\mathrm{R}}}=\Psi_{-}^{\hat{1}}, \quad e^{i \phi_{2}} e^{i Q_{\mathrm{R}}}=\Psi_{-}^{\hat{2}}, \quad e^{-i \phi_{1}} e^{-i P_{\mathrm{R}}}=\Psi_{-}^{\hat{3}}, \quad e^{i \phi_{1}} e^{i P_{\mathrm{R}}}=\Psi_{-}^{\hat{4}} .
\end{aligned}
$$

The introduction of the non-local phases is necessary in order to ensure gauge invariance of the fermions that have their origin in the ungauged theory. This is the same reason for the similar non-local phases appearing in the definitions of the classical parafermions. Since the fermions anticommute we should have included a co-cycle factor in the above definitions. This is not necessary as long as, in the comparison with the supersymmetric $\sigma$-model results of the deformation below, we keep the fermions of the compact coset $\Psi_{ \pm}^{3,4}$ to the left of the non-compact counterparts $\Psi_{ \pm}^{1,2}$. In this manner the effect of the non-trivial co-cycles in the semiclassical limit we are interested in is properly taken into account by the fact that in (3.40) we have $C_{0, k}^{(a)}=1$ for $a=1,2,4$ and $C_{0, k}^{(3)}=-1$ in that limit.

The idea now is to write the complete $\sigma$ model for the deformed background, perform the field redefinitions (4.8) to render the fermions free in the undeformed $\sigma$ model, and consider the leading correction. The discussion will be more transparent if we consider separately the purely bosonic piece, the 2 -fermi term, and the 4 -fermi term. The purely bosonic piece is

$$
S_{\mathrm{bose}}=\int d^{2} \sigma \frac{1}{2}\left(e_{+}^{\hat{1}} e_{-}^{\hat{2}}+e_{-}^{\hat{1}} e_{+}^{\hat{2}}+e_{+}^{\hat{3}} e_{-}^{\hat{4}}+e_{-}^{\hat{3}} e_{+}^{\hat{4}}\right)
$$

and performing the redefinitions (4.8) on this term does not generate any extra bosonic terms. The order $\epsilon$ correction in (4.13) can be written very compactly if we notice that the chiral coframes $e_{ \pm}^{\hat{\imath}}$ with $\hat{\imath}=3,4$, which are the only ones contributing to the correction, can be expressed in terms of the parafermion fields (2.22) and (2.23) as ${ }^{4}$

$$
\begin{aligned}
& e_{+}^{\hat{3}}=e^{i \phi_{1}} \psi+\epsilon \frac{e^{-2 i \omega}}{\cosh ^{2} \rho} e^{-i \phi_{1}} \psi^{\dagger}, \quad e_{-}^{\hat{3}}=e^{-i \phi_{1}} \bar{\psi}+\epsilon \frac{e^{-2 i \omega}}{\cosh ^{2} \rho} e^{i \phi_{1}} \bar{\psi}^{\dagger}, \\
& e_{+}^{\hat{4}}=e^{-i \phi_{1}} \psi^{\dagger}+\epsilon \frac{e^{2 i \omega}}{\cosh ^{2} \rho} e^{i \phi_{1}} \psi, \quad e_{-}^{\hat{4}}=e^{i \phi_{1}} \bar{\psi}^{\dagger}+\epsilon \frac{e^{2 i \omega}}{\cosh ^{2} \rho} e^{-i \phi_{1}} \bar{\psi} .
\end{aligned}
$$

Consequently, the order $\epsilon$ bosonic correction reads:

$$
\delta S_{\text {bose }}=\epsilon \int d^{2} \sigma \frac{k}{\cosh ^{2} \rho}\left(e^{2 i \omega} \psi \bar{\psi}+e^{-2 i \omega} \psi^{\dagger} \bar{\psi}^{\dagger}\right) .
$$

Taking into account (2.35) and (4.12) we see that this is indeed the first line of the perturbation (3.43) based on CFT considerations, with infinitesimal parameter $\epsilon$. Of course it also reproduces the change in the metric (4.3) above, due to the deformation. This matching of

\footnotetext{
${ }^{4}$ Left-/right-moving fields labeled by $+/-$ correspond to holomorphic/antiholomorphic objects.
} 
the bosonic part of the action corresponding to the deformation was shown in [17]. We move on now to the fermionic pieces of the action.

The original 2-fermi terms are ${ }^{5}$

$$
\begin{aligned}
& S_{2-\text { fermi }}=i \int d^{2} \sigma\left(\left(\Psi_{+}^{\hat{1}} \Psi_{+}^{\hat{2}} \omega_{-\hat{2}}^{\hat{2}}+\Psi_{-}^{\hat{1}} \Psi_{-}^{\hat{2}} \omega_{+\hat{2}}^{\hat{2}}\right)+\left(\Psi_{+}^{\hat{3}} \Psi_{+}^{\hat{4}} \omega_{-\hat{4}}^{\hat{4}}+\Psi_{-}^{\hat{3}} \Psi_{-}^{\hat{4}} \omega_{+\hat{4}}^{\hat{4}}\right)+\right. \\
&\left.+\left(\Psi_{+}^{\hat{2}} \Psi_{+}^{\hat{4}} \omega_{-\hat{4}}^{\hat{1}}+\Psi_{-}^{\hat{2}} \Psi_{-}^{\hat{4}} \omega_{+\hat{4}}^{\hat{1}}\right)+\left(\Psi_{+}^{\hat{1}} \Psi_{+}^{\hat{3}} \omega_{-\hat{3}}^{\hat{2}}+\Psi_{-}^{\hat{1}} \Psi_{-}^{\hat{3}} \omega_{+\hat{3}}^{\hat{2}}\right)\right)
\end{aligned}
$$

and the order $\epsilon$ correction they yield is

$$
\begin{aligned}
i \epsilon \int d^{2} \sigma & \frac{1}{\cosh ^{2} \rho}\left[\operatorname { t a n } \theta \left(\Psi_{+}^{\hat{3}} \Psi_{+}^{\hat{4}}\left(e^{2 i \omega-i \varphi-i \phi_{1}} \bar{\psi}-e^{-2 i \omega+i \varphi+i \phi_{1}} \bar{\psi}^{\dagger}\right)\right.\right. \\
& \left.+\Psi_{-}^{\hat{3}} \Psi_{-}^{\hat{4}}\left(e^{2 i \omega-i \varphi+i \phi_{1}} \psi-e^{-2 i \omega+i \varphi-i \phi_{1}} \psi^{\dagger}\right)\right) \\
& +2 \tanh \rho\left(\Psi_{+}^{\hat{2}} \Psi_{+}^{\hat{4}} e^{-3 i \omega+i \phi_{1}} \bar{\psi}^{\dagger}+\Psi_{+}^{\hat{1}} \Psi_{+}^{\hat{3}} e^{3 i \omega-i \phi_{1}} \bar{\psi}\right. \\
& \left.\left.+\Psi_{-}^{\hat{2}} \Psi_{-}^{\hat{4}} e^{-3 i \omega-i \phi_{1}} \psi^{\dagger}+\Psi_{-}^{\hat{1}} \Psi_{-}^{\hat{3}} e^{3 i \omega+i \phi_{1}} \psi\right)\right]
\end{aligned}
$$

In addition to those terms, we obtain extra 2-fermi terms at order $\epsilon$ after applying (4.8) to (4.15). They are

$$
-i \epsilon \int d^{2} \sigma \frac{\tan \theta}{\cosh ^{2} \rho}\left(e^{2 i \omega-i \varphi+i \phi_{1}} \psi \Psi_{-}^{\hat{3}} \Psi_{-}^{\hat{4}}+e^{2 i \omega-i \varphi-i \phi_{1}} \bar{\psi} \Psi_{+}^{\hat{3}} \Psi_{+}^{\hat{4}}\right)+\text { c.c. }
$$

and therefore they cancel the terms in the first two lines of (4.17). All in all the order $\epsilon$ correction to the 2 -fermion terms is

$$
\begin{aligned}
\delta S_{2-\text { fermi }}=2 i \epsilon \int d^{2} \sigma & \frac{\sinh \rho}{\cosh ^{3} \rho}\left(\Psi_{+}^{\hat{2}} \Psi_{+}^{\hat{4}} e^{-3 i \omega+i \phi_{1}} \bar{\psi}^{\dagger}+\Psi_{-}^{\hat{2}} \Psi_{-}^{\hat{4}} e^{-3 i \omega-i \phi_{1}} \psi^{\dagger}\right. \\
& \left.+\Psi_{+}^{\hat{1}} \Psi_{+}^{\hat{3}} e^{3 i \omega-i \phi_{1}} \bar{\psi}+\Psi_{-}^{\hat{1}} \Psi_{-}^{\hat{3}} e^{3 i \omega+i \phi_{1}} \psi\right) .
\end{aligned}
$$

Again, taking into account (2.35) and (4.12) we see that this is the second line of the perturbation (3.43) based on CFT considerations, with the same infinitesimal parameter $\epsilon$.

Finally we consider the 4-fermi terms. The original ones, using the antisymmetry properties of the Riemann tensor to reduce the number of terms, read:

$$
\begin{aligned}
S_{4-\text { fermi }}=2 \int d^{2} \sigma & {\left[R_{\hat{1} \hat{2} \hat{1} \hat{2}} \Psi_{+}^{\hat{1}} \Psi_{+}^{\hat{2}} \Psi_{-}^{\hat{1}} \Psi_{-}^{\hat{2}}+R_{\hat{3} \hat{4} \hat{3} \hat{4}} \Psi_{+}^{\hat{3}} \Psi_{+}^{\hat{4}} \Psi_{-}^{\hat{3}} \Psi_{-}^{\hat{4}}+R_{\hat{2} \hat{4} \hat{2} \hat{4}} \Psi_{+}^{\hat{2}} \Psi_{+}^{\hat{4}} \Psi_{-}^{\hat{2}} \Psi_{-}^{\hat{4}}\right.} \\
& +R_{\hat{1} \hat{3} \hat{1} \hat{3}} \Psi_{+}^{\hat{1}} \Psi_{+}^{\hat{3}} \Psi_{-}^{\hat{1}} \Psi_{-}^{\hat{3}}+R_{\hat{2} \hat{4} \hat{3} \hat{4}}\left(\Psi_{+}^{\hat{2}} \Psi_{+}^{\hat{4}} \Psi_{-}^{\hat{3}} \Psi_{-}^{\hat{4}}+\Psi_{+}^{\hat{3}} \Psi_{+}^{\hat{4}} \Psi_{-}^{\hat{2}} \Psi_{-}^{\hat{4}}\right) \\
& \left.+R_{\hat{1} \hat{3} \hat{3} \hat{4}}\left(\Psi_{+}^{\hat{1}} \Psi_{+}^{\hat{3}} \Psi_{-}^{\hat{3}} \Psi_{-}^{\hat{4}}+\Psi_{+}^{\hat{3}} \Psi_{+}^{\hat{4}} \Psi_{-}^{\hat{1}} \Psi_{-}^{\hat{3}}\right)\right]
\end{aligned}
$$

\footnotetext{
${ }^{5}$ Notice that we have used symmetry properties like $\omega_{-\hat{1}}^{\hat{1}}=-\omega_{-\hat{2}}^{\hat{2}}$ etc. to reduce the number of terms.
} 
To order $\epsilon$ their contribution to the Lagrangian density is

$$
\begin{aligned}
\frac{2 \epsilon}{k \cosh ^{2} \rho} & {\left[-\tan ^{2} \theta \cos 2(\omega-\varphi) \Psi_{+}^{\hat{3}} \Psi_{+}^{\hat{4}} \Psi_{-}^{\hat{3}} \Psi_{-}^{\hat{4}}+\left(\left(1-2 \sinh ^{2} \rho\right) e^{-4 i \omega} \Psi_{+}^{\hat{2}} \Psi_{+}^{\hat{4}} \Psi_{-}^{\hat{2}} \Psi_{-}^{\hat{4}}+\text { c.c. }\right)\right.} \\
& \left.+\left(\tan \theta \tanh \rho e^{-3 i \omega+i \varphi}\left(\Psi_{+}^{\hat{2}} \Psi_{+}^{\hat{4}} \Psi_{-}^{\hat{3}} \Psi_{-}^{\hat{4}}+\Psi_{+}^{\hat{3}} \Psi_{+}^{\hat{4}} \Psi_{-}^{\hat{2}} \Psi_{-}^{\hat{4}}\right)+\text { c.c. }\right)\right] .
\end{aligned}
$$

We obtain also 4-fermion terms at the same order in $\epsilon$ by applying (4.8) to (4.15) and to (4.16). That obtained from (4.15) read:

$$
-2 \epsilon \int d^{2} \sigma \frac{\tan ^{2} \theta \cos 2(\omega-\varphi)}{k \cosh ^{2} \rho} \Psi_{+}^{\hat{3}} \Psi_{+}^{\hat{4}} \Psi_{-}^{\hat{3}} \Psi_{-}^{\hat{4}}
$$

whereas those coming from (4.16) are

$$
\begin{aligned}
2 \epsilon \int d^{2} \sigma & {\left[2 \frac{\tan ^{2} \theta \cos 2(\omega-\varphi)}{k \cosh ^{2} \rho} \Psi_{+}^{\hat{3}} \Psi_{+}^{\hat{4}} \Psi_{-}^{\hat{3}} \Psi_{-}^{\hat{4}}\right.} \\
& \left.-\frac{\tanh \rho \tan \theta}{k \cosh ^{2} \rho} e^{-3 i \omega+i \varphi}\left(\Psi_{+}^{\hat{2}} \Psi_{+}^{\hat{4}} \Psi_{-}^{\hat{3}} \Psi_{-}^{\hat{4}}+\Psi_{+}^{\hat{3}} \Psi_{+}^{\hat{4}} \Psi_{-}^{\hat{2}} \Psi_{-}^{\hat{4}}+\text { c.c. }\right)\right] .
\end{aligned}
$$

Combining everything together we conclude that the total deformation of the 4-fermion terms to leading order in $\epsilon$ is

$$
\delta S_{4-\text { fermi }}=2 \epsilon \int d^{2} \sigma \frac{1-2 \sinh ^{2} \rho}{k \cosh ^{2} \rho}\left(e^{4 i \omega} \Psi_{+}^{\hat{1}} \Psi_{+}^{\hat{3}} \Psi_{-}^{\hat{1}} \Psi_{-}^{\hat{3}}+e^{-4 i \omega} \Psi_{+}^{\hat{2}} \Psi_{+}^{\hat{4}} \Psi_{-}^{\hat{2}} \Psi_{-}^{\hat{4}}\right)
$$

Using (2.35) and (4.12) we see that this is the last line of the perturbation (3.43) based on CFT considerations, with the infinitesimal parameter $\epsilon$.

This completes our proof that the conjectured perturbation (3.43) based on holography and exact CFT considerations completely reproduces the one obtained from the $\sigma$-model semiclassical approach.

\subsection{General deformations of circular NS5-brane distribution}

We analyze now general deformations of the circular NS5-brane system corresponding to small changes of the radius $r_{0} \rightarrow r_{0}+\delta r_{0}$. We can expand $\delta r_{0}$ in Fourier modes $\delta r_{0}=$ $\epsilon r_{0} \cos (n \psi)$ with $n \in \mathbb{Z}$ and focus on each mode separately. The corresponding distribution describes NS5-branes smeared on the $x^{8}-x^{9}$ plane along the curve

$$
\left(x^{8}\right)^{2}+\left(x^{9}\right)^{2}=r_{0}^{2}+\xi(\psi), \quad \psi=\tan ^{-1}\left(x^{9} / x^{8}\right)
$$

which represents a general planar deformation of the circular distribution. To linear order in the deformation parameter we take $\xi(\psi)=2 \epsilon r_{0}^{2} \cos (n \psi)$ and this is the continuous limit of the discrete distribution (3.46) upon the identification $2(j+1) \equiv n$ and for the values of $n$ where $0 \leqslant j \leqslant(k-2) / 2$. The mode with $n=0$, i.e. a uniform rescaling of the radius of the 
circle, corresponds according to the discussion in Sec. 3.2.2 to the operator $j=(k-2) / 2$. Since the latter is of order $k$ the discussion here is not appropriate for that operator and instead we refer the reader to Sec. 3.2.2 where we studied its semiclassical expression. The mode with $n=1$ is not captured from the set of operators under consideration since the corresponding value of $j=-\frac{1}{2}$ does not belong to the allowed interval. In any case, we see that only a finite number of continuous deformations have discrete analogues. These will be the deformations we will be interested in and hence we will assume that $2 \leqslant n \ll k$. The discrete distribution entails also a non-trivial angular distribution $\lambda(\psi)$ and hence the full NS5-brane density reads:

$$
\rho(\mathbf{x})=\frac{1}{\pi} \lambda(\psi) \delta\left(\left(x^{8}\right)^{2}+\left(x^{9}\right)^{2}-r_{0}^{2}-\xi(\psi)\right) \delta\left(x^{6}\right) \delta\left(x^{7}\right),
$$

where $\lambda(\psi)$ is the angular density in (3.48). According to this it is convenient to parameterize the Cartesian coordinates along the curve as

$$
x^{8}=r_{0} \cos \psi(1+\epsilon \cos n \psi), \quad x^{9}=r_{0} \sin \psi(1+\epsilon \cos n \psi),
$$

to linear order in $\epsilon{ }^{6}$ The deformation is geometrically depicted in Figs. 1 and 2.
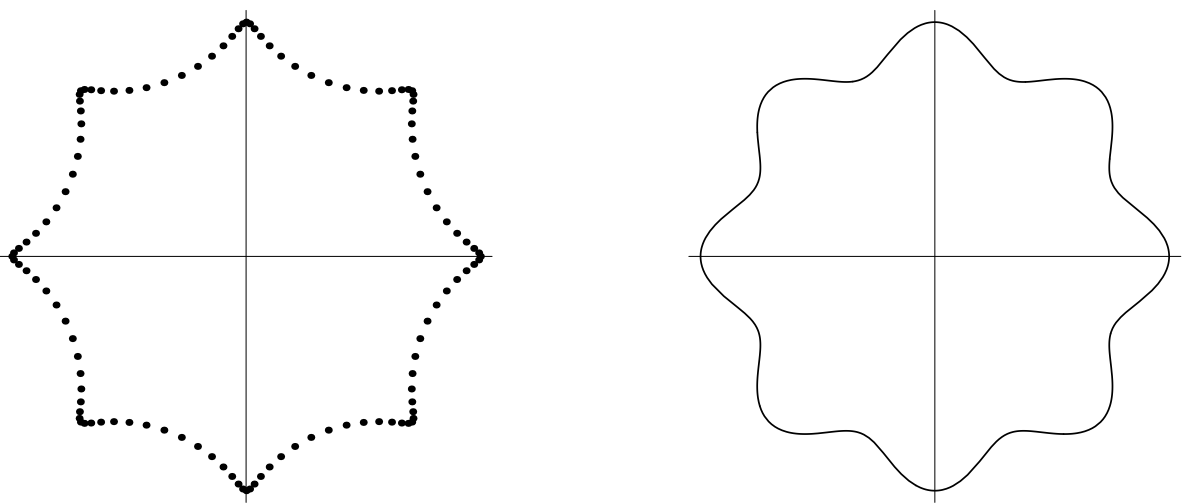

Figure 1: On the left, the discrete distribution of $k \sim 120$ branes on a deformed circle for $n=8$ $(j=3)$ and $\epsilon=0.1$. It is reproduced using (3.46), which in the continuum is equivalent to the parametrization of the deformation described in footnote 6 . On the right, we depict the same deformation in the continuous approximation using the parametrization (4.27).

Using the above, the harmonic function (2.4), in the near-horizon limit where the 1 is dropped, reads:

$$
\begin{aligned}
& H_{n}(\rho, \theta, \psi)=\frac{k}{2 \pi} \\
& \int_{0}^{2 \pi} d \psi^{\prime} \frac{\lambda\left(\psi^{\prime}\right)}{r^{2}+r_{0}^{2}+r_{0}^{2} \sin ^{2} \theta+\xi\left(\psi^{\prime}\right)-2 r_{0} \sqrt{r^{2}+r_{0}^{2}} \sqrt{r_{0}^{2}+\xi\left(\psi^{\prime}\right)} \sin \theta \cos \left(\psi-\psi^{\prime}\right)} .
\end{aligned}
$$

\footnotetext{
${ }^{6}$ An alternative, equivalent to $(3.47)$, coordinate system is the one corresponding to $x^{8}=r_{0}(\cos \phi+$ $\epsilon \cos (n-1) \phi), x^{9}=r_{0}(\sin \phi-\epsilon \sin (n-1) \phi)$, with the angular relation, again to linear order in $\epsilon, \psi=$ $\phi-\epsilon \sin n \phi$. In this system the angular distribution is easily seen to be uniform, i.e. $\lambda(\psi) d \psi=d \phi$. This is the coordinate system used in [17] for $n=2$.
} 
We are interested in the leading order $\epsilon$ correction to $H$ which is given by

$$
\delta H_{n}=\epsilon \frac{k}{2 \pi} \int_{0}^{2 \pi} d \psi^{\prime}\left(\frac{(n-1) \cos n \psi^{\prime}}{A-B \cos \left(\psi^{\prime}-\psi\right)}+\frac{\left(A-2 r_{0}^{2}\right) \cos n \psi^{\prime}}{\left(A-B \cos \left(\psi^{\prime}-\psi\right)\right)^{2}}\right),
$$

where we defined for convenience

$$
A(\rho, \theta)=r^{2}+r_{0}^{2}+r_{0}^{2} \sin ^{2} \theta, \quad B(\rho, \theta)=2 r_{0} \sqrt{r^{2}+r_{0}^{2}} \sin \theta .
$$

Shifting the integration variable as $\psi^{\prime} \rightarrow \psi^{\prime}+\psi$ enables us to write the previous expression in terms of the integral

$$
I_{n}(A, B)=\frac{1}{2 \pi} \int_{0}^{2 \pi} d \psi^{\prime} \frac{\cos n \psi^{\prime}}{A-B \cos \psi^{\prime}}=\frac{1}{\sqrt{A^{2}-B^{2}}}\left(\frac{A-\sqrt{A^{2}-B^{2}}}{B}\right)^{n},
$$

as

$$
\delta H_{n}=\epsilon k \cos n \psi\left((n-1) I_{n}(A, B)-\left(A-2 r_{0}^{2}\right) \frac{\partial}{\partial A} I_{n}(A, B)\right),
$$

where we have used the fact that the same integral as $I_{n}$ but with the cosine replaced by sine vanishes due to its parity. Explicitly, the final result is

$$
\delta H_{n}=\epsilon k \cos n \psi\left(\frac{r_{0} \sin \theta}{\sqrt{r^{2}+r_{0}^{2}}}\right)^{n} \frac{n r^{2}\left(2 r^{2}+r_{0}^{2}\right)-r_{0}^{4}-r_{0}^{2}\left((2-n) r^{2}+r_{0}^{2}\right) \cos 2 \theta}{\left(r^{2}+r_{0}^{2} \cos ^{2} \theta\right)^{3}} .
$$

The deformation of the harmonic function above yields immediately the corresponding deformation of the geometry of the transverse space and the full metric becomes

$$
\begin{aligned}
\frac{d s^{2}}{k}= & \left(\frac{d r^{2}}{r^{2}+r_{0}^{2}}+d \theta^{2}+\frac{r^{2} \cos ^{2} \theta d \tau^{2}}{r^{2}+r_{0}^{2} \cos ^{2} \theta}+\frac{\left(r^{2}+r_{0}^{2}\right) \sin ^{2} \theta d \psi^{2}}{r^{2}+r_{0}^{2} \cos ^{2} \theta}\right)[1+\epsilon \times \\
& \left.\times \cos n \psi\left(\frac{r_{0} \sin \theta}{\sqrt{r^{2}+r_{0}^{2}}}\right)^{n} \frac{n r^{2}\left(2 r^{2}+r_{0}^{2}\right)-r_{0}^{4}-r_{0}^{2}\left((2-n) r^{2}+r_{0}^{2}\right) \cos 2 \theta}{\left(r^{2}+r_{0}^{2} \cos ^{2} \theta\right)^{3}}\right] .
\end{aligned}
$$

Recall, however, that the holographic approach refers to the T-dual of the original geometry and it is necessary to know also the deformation of the $B$ field in order to T-dualize. Instead of following this route, we will start from the holographic result for the bosonic deformation and show that after T-duality and appropriate reparameterization yields exactly the deformation of the transverse metric induced by (4.32). Subsequently, we will verify that the fermionic deformations of the $\mathcal{N}=1 \sigma$ model match the holographic predictions.

The first line of $(3.52)$ is the purely bosonic deformation of the $S L(2, \mathbb{R}) / U(1) \times S U(2) / U(1)$ theory

$$
\delta S_{\text {bose }}=\epsilon \int d^{2} \sigma \frac{k \sin ^{n-2} \theta}{\cosh ^{n} \rho}\left(e^{i(n \omega-(n-2) \varphi)} \psi \bar{\psi}+\text { c.c. }\right)
$$

which implies a corresponding deformation of the metric

$$
d s_{n}^{2}=2 \epsilon k \frac{\sin ^{n-2} \theta}{\cosh ^{n} \rho}\left[\cos n(\omega-\varphi)\left(d \theta^{2}-\tan ^{2} \theta d \varphi^{2}\right)+2 \sin n(\omega-\varphi) \tan \theta d \varphi d \theta\right] .
$$


Explicitly the full metric becomes

$$
\begin{aligned}
\frac{d s^{2}}{k}= & d \rho^{2}+\operatorname{coth}^{2} \rho d \omega^{2}+d \theta^{2}+\tan ^{2} \theta d \varphi^{2}+2 \epsilon \frac{\sin ^{n-2} \theta}{\cosh ^{n} \rho} \times \\
& \times\left(\cos n(\omega-\varphi)\left(d \theta^{2}-\tan ^{2} \theta d \varphi^{2}\right)+2 \sin n(\omega-\varphi) \tan \theta d \varphi d \theta\right) .
\end{aligned}
$$

The dilaton is not modified and hence we can read it from (2.29)

$$
e^{-2 \Phi}=\sinh ^{2} \rho \cos ^{2} \theta
$$

while the antisymmetric tensor remains vanishing. As a first check one can indeed verify that the above background is a solution of the equations of motion to leading order in $\epsilon$.

We proceed now with the T-duality. For that purpose we need to perform as before the reparameterizations $\omega=\psi+\tau$ and $\varphi=\tau$ which make manifest the isometry corresponding to shifts of the new coordinate $\tau$. T-dualizing along $\partial_{\tau}$ yields a new metric

$$
\begin{aligned}
\frac{d s^{2}}{k}= & \frac{d r^{2}}{r^{2}+r_{0}^{2}}+d \theta^{2}+\frac{r^{2} \cos ^{2} \theta d \tau^{2}}{r^{2}+r_{0}^{2} \cos ^{2} \theta}+\frac{\left(r^{2}+r_{0}^{2}\right) \sin ^{2} \theta d \psi^{2}}{r^{2}+r_{0}^{2} \cos ^{2} \theta} \\
& +2 \epsilon\left(\frac{r_{0} \sin \theta}{\sqrt{r^{2}+r_{0}^{2}}}\right)^{n}\left\{-\sin n \psi \sin 2 \theta \frac{\left(r^{2}+r_{0}^{2}\right) d \theta d \psi}{r^{2}+r_{0}^{2} \cos ^{2} \theta}\right. \\
& \left.+\cos n \psi\left(d \theta^{2}+\left(\frac{\sin \theta \cos \theta}{r^{2}+r_{0}^{2} \cos ^{2} \theta}\right)^{2}\left(r^{4} d \tau^{2}-\left(r^{2}+r_{0}^{2}\right)^{2} d \psi^{2}\right)\right)\right\}
\end{aligned}
$$

a dilaton

$$
e^{-2 \Phi}=\frac{r^{2}+r_{0}^{2} \cos ^{2} \theta}{r_{0}^{2}}-2 \epsilon \cos n \psi \frac{r^{2}}{r_{0}^{2}}\left(\frac{r_{0} \sin \theta}{\sqrt{r^{2}+r_{0}^{2}}}\right)^{n}
$$

and a two-form potential with non-vanishing components

$$
\begin{aligned}
\frac{B_{\tau \psi}}{k} & =\frac{1}{\Sigma}+\epsilon \cos n \psi \frac{r^{2}}{\Sigma^{2} \cos ^{2} \theta} \frac{r_{0}^{n} \sin ^{n} \theta}{\left(r^{2}+r_{0}^{2}\right)^{\frac{n}{2}+1}}, \\
\frac{B_{\tau \theta}}{k} & =\epsilon \sin n \psi \frac{r^{2}}{\Sigma \sin \theta \cos \theta} \frac{r_{0}^{n} \sin ^{n} \theta}{\left(r^{2}+r_{0}^{2}\right)^{\frac{n}{2}+1}},
\end{aligned}
$$

where we have defined

$$
\Sigma=1+\frac{r^{2} \tan ^{2} \theta}{r^{2}+r_{0}^{2}} .
$$

Finally, if we perform the following reparameterizations

$$
\begin{aligned}
\delta r & =-\frac{\epsilon r}{n-1} \frac{r_{0}^{n} \sin ^{n} \theta}{\left(r^{2}+r_{0}^{2}\right)^{\frac{n}{2}-1}\left(r^{2}+r_{0}^{2} \cos ^{2} \theta\right)} \cos n \psi, \\
\delta \theta & =-\frac{\epsilon \cot \theta}{n-1} \frac{r_{0}^{n} \sin ^{n} \theta}{\left(r^{2}+r_{0}^{2}\right)^{\frac{n}{2}-1}\left(r^{2}+r_{0}^{2} \cos ^{2} \theta\right)} \cos n \psi, \\
\delta \psi & =\frac{\epsilon}{n-1} \frac{1}{\sin ^{2} \theta} \frac{r_{0}^{n} \sin ^{n} \theta}{\left(r^{2}+r_{0}^{2}\right)^{\frac{n}{2}}} \sin n \psi,
\end{aligned}
$$


and set $r=r_{0} \sinh \rho$, we find that the metric in the new coordinate system is exactly the metric (4.33) we found earlier. Note that these reparameterizations do not change the angular character of the variables $\theta$ and $\psi$ since they are nowhere singular and their periodicity is respected.

We proceed now to the analysis of the fermionic terms in the $\mathcal{N}=1 \sigma$ model. The necessary spin-connection and curvature two-form associated to the metric (4.36) are given in App. D. As in the case of the elliptic deformation, we need first perform the redefinitions (4.8) in order to render the $\sigma$-model fermions free. The original order $\epsilon 2$-fermi terms are

$$
\begin{aligned}
i \epsilon \int d^{2} \sigma & \frac{\sin ^{n-2} \theta}{\cosh ^{n} \rho}\left[\operatorname { t a n } \theta \left(\Psi_{+}^{\hat{3}} \Psi_{+}^{\hat{4}}\left(e^{n i(\omega-\varphi)+i \varphi-i \phi_{1}} \bar{\psi}-e^{-n i(\omega-\varphi)-i \varphi+i \phi_{1}} \bar{\psi}^{\dagger}\right)\right.\right. \\
& \left.+\Psi_{-}^{\hat{3}} \Psi_{-}^{\hat{4}}\left(e^{n i(\omega-\varphi)+i \varphi+i \phi_{1}} \psi-e^{-n i(\omega-\varphi)-i \varphi-i \phi_{1}} \psi^{\dagger}\right)\right) \\
& +n \tanh \rho\left(\Psi_{+}^{\hat{2}} \Psi_{+}^{\hat{4}} e^{-n i(\omega-\varphi)-2 i \varphi-i \omega+i \phi_{1}} \bar{\psi}^{\dagger}+\Psi_{+}^{\hat{1}} \Psi_{+}^{\hat{3}} e^{n i(\omega-\varphi)+2 i \varphi+i \omega-i \phi_{1}} \bar{\psi}\right. \\
& \left.\left.+\Psi_{-}^{\hat{2}} \Psi_{-}^{\hat{4}} e^{-n i(\omega-\varphi)-2 i \varphi-i \omega-i \phi_{1}} \psi^{\dagger}+\Psi_{-}^{\hat{1}} \Psi_{-}^{\hat{3}} e^{n i(\omega-\varphi)+2 i \varphi+i \omega+i \phi_{1}} \psi\right)\right]
\end{aligned}
$$

and, along with the contribution of (4.34) from the field redefinition (4.8), combine to

$$
\begin{aligned}
\delta S_{2-\text { fermi }}=i \epsilon n \int d^{2} \sigma & \frac{\sin ^{n-2} \theta \sinh \rho}{\cosh ^{3} \rho}\left(\Psi_{+}^{\hat{2}} \Psi_{+}^{\hat{4}} e^{-n i(\omega-\varphi)-2 i \varphi-i \omega+i \phi_{1}} \bar{\psi}^{\dagger}\right. \\
& +\Psi_{-}^{\hat{2}} \Psi_{-}^{\hat{4}} e^{-n i(\omega-\varphi)-2 i \varphi-i \omega-i \phi_{1}} \psi^{\dagger}+\Psi_{+}^{\hat{1}} \Psi_{+}^{\hat{3}} e^{n i(\omega-\varphi)+2 i \varphi+i \omega-i \phi_{1}} \bar{\psi} \\
& \left.+\Psi_{-}^{\hat{1}} \Psi_{-}^{\hat{3}} e^{n i(\omega-\varphi)+2 i \varphi+i \omega+i \phi_{1}} \psi\right) .
\end{aligned}
$$

This matches the operators in the second line of (3.52) that we computed using the conjectured holography and CFT.

Let us finally check the 4-fermion terms. Besides the ones coming from the original 4-fermi interactions we have additional ones coming from (4.34) and from the original 2-fermion terms after performing the field redefinition (4.8). Combining everything together results in the expression

$$
\begin{aligned}
\delta S_{4-\text { fermi }}=\epsilon n \int d^{2} \sigma & \frac{\left(1-n \sinh ^{2} \rho\right) \sin ^{n-2} \theta}{k \cosh ^{n} \rho}\left(e^{i n(\omega-\varphi)+2 i \omega+2 i \varphi} \Psi_{+}^{\hat{1}} \Psi_{+}^{\hat{3}} \Psi_{-}^{\hat{1}} \Psi_{-}^{\hat{3}}\right. \\
& \left.+e^{-i n(\omega-\varphi)-2 i \omega-2 i \varphi} \Psi_{+}^{\hat{2}} \Psi_{+}^{\hat{4}} \Psi_{-}^{\hat{2}} \Psi_{-}^{\hat{4}}\right)
\end{aligned}
$$

which matches exactly the last line of (3.52).

\section{Summary and perspectives}

The starting point of our analysis was an exact $\mathcal{N}=4$ string background (metric, antisymmetric tensor and dilaton), generated by $k$ parallel NS5-branes distributed over a circle 
transverse to their worldvolume. In the near-horizon regime it is described by the KazamaSuzuki model $S U(2) / U(1) \times S L(2, \mathbb{R}) / U(1)$.

The geometric deformations of this setup can be studied in two different manners. Either by using holography, which relates NS5-brane worldvolume operators to $\sigma$-model worldsheet operators (Sec. 3), or by explicitly perturbing the locus of the NS5-branes, from a circle to an $n$-bump deformed circle, and computing the corresponding $\sigma$-model background fields (Sec. 4). From the latter we can read-off the worldsheet operators that trigger the perturbation. These are marginal since the NS5-brane perturbations do not alter the conformality of the $\mathcal{N}=4$ string backgrounds at hand. Actually, they exactly marginal as they originate from chiral primary operators. They are indeed described in terms of compact parafermions dressed with non-compact and compact primaries, whose conformal weights add up to $(1,1)$. All these generalize the results obtained in [17] for the elliptical deformation of the circle, to the infinite tower of $n$-bump modes. As already stressed, these computations are performed in the semiclassical approximation, but the whole framework allows to safely conjecture their validity beyond that level.

The marginal worldsheet operators that are revealed by the deformed- $\sigma$-model approach turn out to be in agreement with those found using the LST dictionary for $n$-bump NS5brane displacements away from the circle. This brilliantly demonstrates the validity of the holographic duality for a large class of operators. To the best of our knowledge, this result is unique as is the construction of the set of non-left-right-factorized marginal operators of the $S U(2) / U(1) \times S L(2, \mathbb{R}) / U(1)$ conformal model.

One could generalize this study in a variety of directions. For instance, one can consider non-planar deformations of the NS5-branes and verify the validity of the holographic dictionary. Furthermore, it would be very interesting to extend this analysis to time-dependent deformations where issues like supersymmetry breaking can be addressed in an exact CFT framework. A challenging open problem pertains as to the exact CFT description of NS5brane systems, for instance the elliptic distribution, which preserve a large part of the original transverse-space symmetries. Presumably generalizations of the compact and non-compact parafermions would be instrumental for such an endeavor.

\section{Acknowledgements}

The authors would like to thank their colleagues C. Bachas, C. Kounnas and V. Niarchos for stimulating exchanges. The work of Angelos Fotopoulos is partially supported by the European Community's Human Potential Programme under contract MRTN-CT-2004-005104 and by the Italian MUR under contracts PRIN-2005023102 and PRIN-2005024045. Angelos Fotopoulos would like to thank CERN Theory Division for its warm hospitality where part of this work has been done. Marios Petropoulos thanks Neuchâtel University, Patras University and CERN Theory Division for kind hospitality at various stages of this collaboration, and acknowledges financial support by the Swiss National Science Foundation, 
the French Agence Nationale pour la Recherche, contract 05-BLAN-0079-01, and the EU under the contracts MEXT-CT-2003-509661, MRTN-CT-2004-005104 and MRTN-CT-2004503369. Nikolaos Prezas wishes to thank the Ecole Polytechnique for its warm hospitality where part of this work was done. Konstadinos Sfetsos acknowledges partial support provided through the European Community's program "Constituents, Fundamental Forces and Symmetries of the Universe" with contract MRTN-CT-2004-005104, the INTAS contract 0351-6346 "Strings, branes and higher-spin gauge fields" and the Greek Ministry of Education

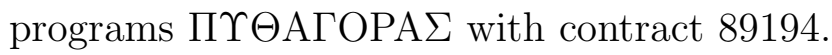

\section{A. Linear-dilaton background and the $\mathcal{N}=4$ superconformal alge- bra}

The CHS conformal field theory $\mathbb{R}_{\phi} \times S U(2)_{k}$ contains a linear dilaton along the $\phi$ direction

with background charge $q=\sqrt{\frac{2}{k}}$ (where $k$ is the number of NS5-branes) and a $\mathcal{N}=1$ supersymmetric $S U(2)$ WZW model at level $k$. We will exhibit the $\mathcal{N}=4$ superconformal algebra [40] supported on $\mathbb{R}_{\phi} \times S U(2)_{k}$ following the discussion of [8].

The supersymmetric WZW model is decomposed into a bosonic $S U(2)$ WZW model at level $k-2$ with affine currents $J^{i}$ and three free fermions $\psi_{i}, i=1,2,3$. We denote by $\phi$ the boson corresponding to the dilaton direction and by $\psi_{\phi}$ is its superpartner. The operator algebra is

$$
\begin{aligned}
\partial \phi(z) \partial \phi(w) & =-\frac{1}{(z-w)^{2}}, \\
\psi_{i}(z) \psi_{j}(w) & =\frac{\delta_{i j}}{z-w}, \\
J^{i}(z) J^{j}(w) & =\frac{(k-2) \delta_{i j}}{2(z-w)^{2}}+i \epsilon^{i j k} \frac{J^{k}(w)}{z-w},
\end{aligned}
$$

with $\epsilon^{123}=1$. We can change basis to $J^{ \pm}=J^{1} \pm i J^{2}$ and the corresponding OPEs read:

$$
\begin{aligned}
J^{3}(z) J^{3}(w) & =\frac{k-2}{2(z-w)^{2}}, \\
J^{3}(z) J^{ \pm}(w) & = \pm \frac{J^{ \pm}(w)}{z-w} \\
J^{+}(z) J^{-}(w) & =\frac{k-2}{(z-w)^{2}}+\frac{2 J^{3}(w)}{z-w} .
\end{aligned}
$$

We now define

$$
\begin{aligned}
\psi^{ \pm} & =\frac{1}{\sqrt{2}}\left(\psi_{1} \pm i \psi_{2}\right) \\
\psi & =\frac{1}{\sqrt{2}}\left(\psi_{\phi}+i \psi_{3}\right)
\end{aligned}
$$


and their OPEs read:

$$
\psi(z) \psi^{*}(w)=\psi^{+}(z) \psi^{-}(w)=\frac{1}{z-w} .
$$

The currents of the supersymmetric $S U(2)$ WZW model at level $k$ are given by

$$
J_{i}^{\mathrm{tot}}=J_{i}-\frac{i}{2} \epsilon_{i j k} \psi_{j} \psi_{k}
$$

and in particular

$$
J_{3}^{\text {tot }}=J_{3}-i \psi_{1} \psi_{2}=J_{3}+\psi^{+} \psi^{-} .
$$

The primaries $\Phi_{j ; m, \bar{m}}^{s u}$ of the bosonic $S U(2)$ WZW model have conformal weight

$$
h=\frac{j(j+1)}{k},
$$

while those of the linear dilaton theory have

$$
h\left(e^{a \phi}\right)=-\frac{1}{2} a(a+q) .
$$

The system $\mathbb{R}_{\phi} \times S U(2)$ is known to exhibit a "small" $\mathcal{N}=4$ superconformal symmetry, in line with the fact that the dual configuration of NS5-branes is 1/2 BPS and in type II string theories preserves 16 supersymmetries in space-time. The superconformal generators are

$$
\begin{aligned}
G & =i \psi_{\phi} \partial \phi+i q \partial \psi_{\phi}+q\left(J_{1} \psi_{1}+J_{2} \psi_{2}+J_{3} \psi_{3}-i \psi_{1} \psi_{2} \psi_{3}\right), \\
G_{1} & =i \psi_{1} \partial \phi+i q \partial \psi_{1}+q\left(-J_{1} \psi_{\phi}+J_{2} \psi_{3}-J_{3} \psi_{2}+i \psi_{2} \psi_{3} \psi_{\phi}\right), \\
G_{2} & =i \psi_{2} \partial \phi+i q \partial \psi_{2}+q\left(-J_{2} \psi_{\phi}+J_{3} \psi_{1}-J_{1} \psi_{3}+i \psi_{3} \psi_{1} \psi_{\phi}\right), \\
G_{3} & =i \psi_{3} \partial \phi+i q \partial \psi_{3}+q\left(-J_{3} \psi_{\phi}+J_{1} \psi_{2}-J_{2} \psi_{1}+i \psi_{1} \psi_{2} \psi_{\phi}\right)
\end{aligned}
$$

and the $S U(2) R$-symmetry currents are

$$
\begin{aligned}
S_{1} & =-\frac{i}{2}\left(\psi_{\phi} \psi_{1}+\psi_{2} \psi_{3}\right), \\
S_{2} & =-\frac{i}{2}\left(\psi_{\phi} \psi_{2}+\psi_{3} \psi_{1}\right), \\
S_{3} & =-\frac{i}{2}\left(\psi_{\phi} \psi_{3}+\psi_{1} \psi_{2}\right) .
\end{aligned}
$$

These currents generate an $S U(2)$ current algebra at level one. The energy-momentum tensor reads:

$$
T=-\frac{1}{2}(\partial \phi)^{2}-\frac{1}{2} q \partial^{2} \phi+\frac{J^{i} J^{i}}{k}-\frac{1}{2} \psi^{*} \partial \psi-\frac{1}{2} \psi \partial \psi^{*}-\frac{1}{2} \psi^{+} \partial \psi^{-}-\frac{1}{2} \psi^{-} \partial \psi^{+} .
$$

For our purposes it will be useful to exhibit an $\mathcal{N}=2$ subalgebra of the above algebra with $G$ and $G_{3}$ the corresponding superconformal generators. We can define

$$
G^{ \pm}=\frac{1}{\sqrt{2}}\left(G \pm i G_{3}\right)
$$


and explicitly they are given $b y^{7}$

$$
\begin{aligned}
& G^{+}=i \psi\left(\partial \phi-q J_{3}^{\mathrm{tot}}\right)+i q \partial \psi+q J^{-} \psi^{+}, \\
& G^{-}=i \psi^{*}\left(\partial \phi+q J_{3}^{\mathrm{tot}}\right)+i q \partial \psi^{*}+q J^{+} \psi^{-} .
\end{aligned}
$$

The $U(1) R$-symmetry generator is

$$
J_{\mathrm{R}}=\psi \psi^{*}+\psi^{+} \psi^{-}=2 S_{3}
$$

and the level one $S U(2)$ raising and lowering operators are

$$
S^{+}=S_{2}+i S_{1}=\psi \psi^{+}, \quad S^{-}=S_{2}-i S_{1}=\psi^{-} \psi^{*} .
$$

We can also form the combinations

$$
\tilde{G}^{ \pm}=\frac{1}{\sqrt{2}}\left(G_{1} \pm i G_{2}\right)
$$

which explicitly read:

$$
\begin{aligned}
& \tilde{G}^{+}=i \psi^{+}\left(\partial \phi+q\left(J_{3}-\psi \psi^{*}\right)\right)+i q \partial \psi^{+}-q J^{+} \psi, \\
& \tilde{G}^{-}=i \psi^{-}\left(\partial \phi-q\left(J_{3}-\psi \psi^{*}\right)\right)+i q \partial \psi^{-}-q J^{-} \psi^{*} .
\end{aligned}
$$

The operators defined above satisfy the small $\mathcal{N}=4$ superconformal algebra

$$
\begin{aligned}
J_{\mathrm{R}}(z) S^{ \pm}(w) & \sim \pm \frac{2 S^{ \pm}}{z-w}, & S^{+}(z) S^{-}(w) & \sim \frac{J_{\mathrm{R}}(w)}{z-w}+\frac{1}{(z-w)^{2}}, \\
S^{+}(z) G^{-}(w) & \sim-\frac{\tilde{G}^{+}}{z-w}, & S^{+}(z) \tilde{G}^{-}(w) & \sim \frac{G^{+}}{z-w}, \\
S^{-}(z) G^{+}(w) & \sim-\frac{\tilde{G}^{-}}{z-w}, & S^{-}(z) \tilde{G}^{+}(w) & \sim \frac{G^{-}}{z-w} .
\end{aligned}
$$

The OPEs of $G^{ \pm}$and $\tilde{G}^{ \pm}$with $J_{\mathrm{R}}$ imply the charges +1 for $G^{+}, \tilde{G}^{+}$and -1 for $G^{-}, \tilde{G}^{-}$. The remaining OPEs of the $S U(2)$ currents with the supercharges are regular. In addition the doublets have singular OPEs among themselves

$$
\begin{aligned}
G^{+}(z) G^{-}(w) & \sim \frac{2 c}{3(z-w)^{3}}+\frac{2 J_{\mathrm{R}}(w)}{(z-w)^{2}}+\frac{2 T(w)+\partial J_{\mathrm{R}}(w)}{z-w}, \\
\tilde{G}^{+}(z) \tilde{G}^{-}(w) & \sim \frac{2 c}{3(z-w)^{3}}+\frac{2 J_{\mathrm{R}}(w)}{(z-w)^{2}}+\frac{2 T(w)+\partial J_{\mathrm{R}}(w)}{z-w}, \\
G^{+}(z) \tilde{G}^{+}(0) & \sim \frac{S^{+}(w)}{(z-w)^{2}}+\frac{\partial S^{+}(w)}{2(z-w)} \\
G^{-}(z) \tilde{G}^{-}(0) & \sim \frac{S^{-}(w)}{(z-w)^{2}}+\frac{\partial S^{-}(w)}{2(z-w)}
\end{aligned}
$$

\footnotetext{
${ }^{7} \mathrm{An}$ interesting feature of this construction is that although $\partial \phi$ is not a conformal primary field in the linear-dilaton background, the $G^{ \pm}$are primaries since the cubic term coming from contracting $\partial^{2} \phi$ with $\partial \phi$ cancels out another cubic term coming from $\psi \partial \psi^{*}$ or $\psi^{*} \partial \psi$ contracted with either $\partial \psi$ or $\partial \psi^{*}$.
} 
where $c=6$ for the CHS background.

In the remaining part of this appendix we would like to demonstrate that the operators in (3.12), when inserted in (3.2), leave the $\mathcal{N}=4$ algebra unbroken. We follow the analysis of [41]. As it is well-known a perturbation which preserves at least an $\mathcal{N}=2$ should be constructed using chiral or antichiral fields. The chiral fields $O_{a}$ are annihilated by $G_{-\frac{1}{2}}^{+}$and have charge $\mathcal{Q}_{\mathrm{R}}=+1$ and dimension $h=\frac{1}{2}$. The antichiral fields are annihilated by $G_{-\frac{1}{2}}^{-}$ and have charge $\mathcal{Q}_{\mathrm{R}}=-1$ and dimension $h=\frac{1}{2}$. The general perturbation which preserves the $\mathcal{N}=2$ subalgebra containing $G^{-}$and $G^{+}$is

$$
\delta S=\int d^{2} z\left(\lambda_{a} G^{-} O_{a}+\bar{\lambda}_{a} G^{+} \bar{O}_{a}\right)
$$

where $O_{a} / \bar{O}_{a}$ is an chiral/antichiral field and $G^{-} O_{a}$ means $\oint d z G^{-}(z) O_{a}(0)$ (which will be implied for the rest of this appendix). The index $a$ parameterizes the set of chiral (antichiral) operators. Then it turns out that the requirement of $\mathcal{N}=4$ SCFT invariance imposes the additional constraints:

$$
S^{-} O_{a}=M_{a}^{\bar{b}} \bar{O}_{a}, \quad S^{+} \bar{O}_{a}=-M_{\bar{a}}^{b} O_{a}, \quad M_{a}^{\bar{b}} M_{\bar{b}}^{c}=\delta_{a}^{c}
$$

Now we will consider the operators (3.12). In this case the matrix $M_{a}^{\bar{b}}$ is one by one and can be set to unity. Therefore the index $a$ can be dropped and the operator under consideration is

$$
O=\psi^{+} \Phi_{j ; j, j}^{s u} e^{-q(j+1) \phi},
$$

where we write only its holomorphic part. It is easy to verify, using the explicit expressions of the $\mathcal{N}=4$ generators, that it is indeed a chiral operator as claimed in the main text. Moreover, it is straightforward to show that the operator

$$
\bar{O}=S^{-} O=-\psi^{*} \Phi_{j ; j, j}^{s u} e^{-q(j+1) \phi}
$$

is an antichiral operator. Hence the constraints (A.23) are satisfied and perturbing the theory with the upper component of any of the operators (A.24) does not spoil $\mathcal{N}=4$ superconformal invariance. The same discussion can be trivially extended to the antiholomorphic sector.

\section{B. Parafermionic operator products}

\section{B.1 Compact parafermions and $S U(2)$ current algebra}

Let us consider the $S U(2)$ current algebra at level $k \geq 2$ whose currents obey the following 
operator algebra:

$$
\begin{aligned}
J^{3}(z) J^{3}(w) & \sim \frac{k}{2} \frac{1}{(z-w)^{2}}, \\
J^{3}(z) J^{ \pm}(w) & \sim \pm \frac{J^{ \pm}(w)}{z-w}, \\
J^{+}(z) J^{-}(w) & \sim \frac{k}{(z-w)^{2}}+\frac{2 J^{3}(w)}{z-w}
\end{aligned}
$$

and decompose the currents as

$$
\begin{aligned}
J^{3} & =i \sqrt{\frac{k}{2}} \partial P, \\
J^{+} & =\sqrt{k} \psi \exp i \sqrt{\frac{2}{k}} P, \\
J^{-} & =\sqrt{k} \psi^{\dagger} \exp -i \sqrt{\frac{2}{k}} P,
\end{aligned}
$$

with $P$ being a free boson and $\psi, \psi^{\dagger}$ being the basic the parafermion fields.

In general the compact parafermionic algebra at level $k$ contains a set of objects $\psi_{l}$ and $\psi_{l}^{\dagger}=\psi_{-l}$ where $l=0,1, \ldots, k-1$ and so that $\psi_{0}=1$ and $\psi_{1}=\psi$ (see for instance [42]). Their conformal dimensions are $\Delta_{l}=l-\frac{l^{2}}{k}$. The generic parafermion OPEs are

$$
\psi_{l_{1}}(z) \psi_{l_{2}}(w)=C_{l_{1}, l_{2}}(z-w)^{\Delta_{l_{1}+l_{2}}-\Delta_{l_{1}}-\Delta_{l_{2}}}\left(\psi_{l_{1}+l_{2}}(z)+\mathcal{O}(z-w)\right)
$$

and

$$
\psi_{l}(z) \psi_{l}^{\dagger}(w)=(z-w)^{-2 \Delta_{l}}\left(1+\frac{2 \Delta_{l}}{c}(z-w)^{2} T(w)+\mathcal{O}\left((z-w)^{3}\right)\right)
$$

where $c=\frac{2(k-1)}{k+2}$ is the central charge of the parafermion theory, $T$ the corresponding energymomentum tensor and the structure constants $C_{l_{1}, l_{2}}$, which are determined by associativity of the OPE, are given by

$$
C_{l_{1}, l_{2}}=\left(\frac{\Gamma\left(k-l_{1}+1\right) \Gamma\left(k-l_{2}+1\right) \Gamma\left(l_{1}+l_{2}+1\right)}{\Gamma\left(l_{1}+1\right) \Gamma\left(l_{2}+1\right) \Gamma(k+1) \Gamma\left(k-l_{1}-l_{2}+1\right)}\right)^{\frac{1}{2}} .
$$

Using (B.3) and (B.4) we find that

$$
\begin{aligned}
\psi(z) \psi(w) & \sim(z-w)^{-\frac{2}{k}} \psi_{2}(w) \\
\psi(z) \psi^{\dagger}(w) & \sim(z-w)^{-2\left(1-\frac{1}{k}\right)}
\end{aligned}
$$

and upon using also

$$
\begin{aligned}
\partial P(z) \partial P(w) & \sim-\frac{1}{(z-w)^{2}}, \\
e^{i a P(z)} e^{i b P(w)} & \sim(z-w)^{a b} e^{i a P(z)+i b P(w)},
\end{aligned}
$$

we can check that the decomposed currents in (B.2) obey the $S U(2)$ current algebra. 
An affine $S U(2)$ primary $\Phi_{j ; m, \bar{m}}^{s u}$ satisfies

$$
\begin{aligned}
J^{3}(z) \Phi_{j ; m, \bar{m}}^{s u}(w) & \sim \frac{m}{z-w} \Phi_{j ; m, \bar{m}}^{s u}, \\
J^{ \pm}(z) \Phi_{j ; m, \bar{m}}^{s u}(w) & \sim \frac{j \mp m}{z-w} \Phi_{j ; m \pm 1, \bar{m}}^{s u}
\end{aligned}
$$

and decomposing it in terms of the parafermionic primary $\psi_{j ; m, \bar{m}}$ as

$$
\Phi_{j ; m, \bar{m}}^{s u}=\psi_{j ; m, \bar{m}} \exp i\left(m \sqrt{\frac{2}{k}} P_{L}+\bar{m} \sqrt{\frac{2}{k}} P_{R}\right),
$$

leads to the following OPEs for the primaries $\psi_{j ; m, \bar{m}}$ :

$$
\begin{aligned}
\psi(z) \psi_{j ; m, \bar{m}}(w) & \sim \frac{j-m}{\sqrt{k}} \frac{\psi_{j ; m+1, \bar{m}}}{(z-w)^{1+\frac{2 m}{k}}}, \\
\psi^{\dagger}(z) \psi_{j ; m, \bar{m}}(w) & \sim \frac{j+m}{\sqrt{k}} \frac{\psi_{j ; m-1, \bar{m}}}{(z-w)^{1-\frac{2 m}{k}}} .
\end{aligned}
$$

\section{B.2 Non-compact parafermions and $S L(2, \mathbb{R})$ current algebra}

We start now from the $S L(2, \mathbb{R})$ current algebra at level $k \geq 2$

$$
\begin{aligned}
K^{3}(z) K^{3}(w) & \sim-\frac{k}{2} \frac{1}{(z-w)^{2}}, \\
K^{3}(z) K^{ \pm}(w) & \sim \pm \frac{K^{ \pm}(w)}{(z-w)} \\
K^{+}(z) K^{-}(w) & \sim \frac{k}{(z-w)^{2}}-\frac{2 K^{3}(w)}{z-w}
\end{aligned}
$$

and decompose the currents as

$$
\begin{aligned}
K^{3} & =-\sqrt{\frac{k}{2}} \partial P, \\
K^{+} & =\sqrt{k} \pi \exp \sqrt{\frac{2}{k}} P, \\
K^{-} & =\sqrt{k} \pi^{\dagger} \exp -\sqrt{\frac{2}{k}} P,
\end{aligned}
$$

with $P$ is a boson and $\pi, \pi^{\dagger}$ are the fundamental non-compact parafermion fields.

The non-compact parafermionic algebra at level $k$ contains an infinite set of objects $\pi_{l}$ and $\pi_{l}^{\dagger}=\pi_{-l}$ where $l=0,1,2, \ldots$ and so that $\pi_{0}=1$ and $\pi_{1}=\pi$. Their conformal dimensions are $\Delta_{l}=l+\frac{l^{2}}{k}$ and their OPEs are the same as those of the compact parafermions but with the central charge being $c=\frac{2(k+1)}{k-2}$ and the structure constants $C_{l_{1}, l_{2}}$ changed to

$$
C_{l_{1}, l_{2}}=\left(\frac{\Gamma(k) \Gamma\left(k+l_{1}+l_{2}\right) \Gamma\left(l_{1}+l_{2}+1\right)}{\Gamma\left(l_{1}+1\right) \Gamma\left(l_{2}+1\right) \Gamma\left(k+l_{1}\right) \Gamma\left(k+l_{2}\right)}\right)^{\frac{1}{2}} .
$$


The OPEs we will need are

$$
\begin{aligned}
\pi(z) \pi(w) & \sim(z-w)^{\frac{2}{k}} \pi_{2}(w), \\
\pi(z) \pi^{\dagger}(w) & \sim(z-w)^{-2\left(1+\frac{1}{k}\right)} .
\end{aligned}
$$

The $S L(2, \mathbb{R})$ affine primaries $\Phi_{j ; m, \bar{m}}^{s l}$ satisfy

$$
\begin{aligned}
K^{3}(z) \Phi_{j ; m, \bar{m}}^{s l}(w) & =\frac{m}{z-w} \Phi_{j ; m, \bar{m}}^{s l}, \\
K^{ \pm}(z) \Phi_{j ; m, \bar{m}}^{s l}(w) & =\frac{m \pm(j+1)}{z-w} \Phi_{j ; m \pm 1, \bar{m}}^{s l}
\end{aligned}
$$

and decomposing them as

$$
\Phi_{j ; m, \bar{m}}^{s l}=\pi_{j ; m, \bar{m}} \exp \left(m \sqrt{\frac{2}{k}} P_{\mathrm{L}}+\bar{m} \sqrt{\frac{2}{k}} P_{\mathrm{R}}\right),
$$

leads to the following OPEs for the parafermionic primaries $\pi_{j ; m, \bar{m}}$ :

$$
\begin{aligned}
\pi(z) \pi_{j ; m, \bar{m}}(w) & \sim \frac{m+(j+1)}{\sqrt{k}} \frac{\pi_{j ; m+1, \bar{m}}}{(z-w)^{1-\frac{2 m}{k}}}, \\
\pi^{\dagger}(z) \pi_{j ; m, \bar{m}}(w) & \sim \frac{m-(j+1)}{\sqrt{k}} \frac{\pi_{j ; m-1, \bar{m}}}{(z-w)^{1+\frac{2 m}{k}}} .
\end{aligned}
$$

\section{Superconformal $\mathcal{N}=2$ algebras}

Using the decomposition of the $\mathcal{N}=2$ minimal model in terms of the bosonic parafermion theory and a free scalar $P$, we can write an explicit realization of the $\mathcal{N}=2$ superconformal algebra generators. They read:

$$
\begin{aligned}
G^{+s u} & =\sqrt{\frac{2(k-2)}{k}} \psi^{\dagger} \exp -i \sqrt{\frac{k}{k-2}} P_{\mathrm{L}}, \\
G^{-s u} & =\sqrt{\frac{2(k-2)}{k}} \psi \exp +i \sqrt{\frac{k}{k-2}} P_{\mathrm{L}},
\end{aligned}
$$

while the $R$-symmetry $U(1)$ current is

$$
J^{s u}=-i \sqrt{\frac{k-2}{k}} \partial P .
$$

Notice that when the supersymmetric minimal model is at level $k$ the bosonic parafermions are at level $k-2$.

Similarly, for the $\mathcal{N}=2$ Kazama-Suzuki model of $S L(2, \mathbb{R}) / U(1)$ at level $k$ the superconformal generators can be written in terms of the non-compact parafermions at level $k+2$ and of the free scalar $Q$ as

$$
\begin{aligned}
& G^{+s l}=\sqrt{\frac{2(k+2)}{k}} \pi^{\dagger} \exp i \sqrt{\frac{k}{k+2}} Q_{\mathrm{L}}, \\
& G^{-s l}=\sqrt{\frac{2(k+2)}{k}} \pi \exp -i \sqrt{\frac{k}{k+2}} Q_{\mathrm{L}},
\end{aligned}
$$


while the $R$-symmetry $U(1)$ current is

$$
J^{s l}=i \sqrt{\frac{k+2}{k}} \partial Q .
$$

It is straightforward to verify that these generators satisfy the $\mathcal{N}=2$ superconformal algebra by using the OPEs of the parafermion theory provided in App. B.

The superconformal generators of the total $\mathcal{N}=2$ algebra on $S U(2) / U(1) \times S L(2, \mathbb{R}) / U(1)$ are

$$
G^{+}=G^{+s u}+G^{+s l}, \quad G^{-}=G^{-s u}+G^{-s l}
$$

and the total $U(1) R$-current is

$$
J=J^{s u}+J^{s l}
$$

Analogous expressions hold for the antiholomorphic sector.

\section{Coframes, spin connections and curvature two-forms}

The full metric corresponding to small deformations of the circle is given in (4.36) which for reference we copy here

$$
\begin{aligned}
\frac{d s^{2}}{k}= & d \rho^{2}+\operatorname{coth}^{2} \rho d \omega^{2}+d \theta^{2}+\tan ^{2} \theta d \varphi^{2}+2 \epsilon \frac{\sin ^{n-2} \theta}{\cosh ^{n} \rho} \times \\
& \times\left[\cos n(\omega-\varphi)\left(d \theta^{2}-\tan ^{2} \theta d \varphi^{2}\right)+2 \sin n(\omega-\varphi) \tan \theta d \varphi d \theta\right] .
\end{aligned}
$$

The coframe we select is inspired by the form of the classical parafermions in the unperturbed case and it reads:

$$
\begin{aligned}
e^{\hat{1}} & =\sqrt{k}(d \rho-i \operatorname{coth} \rho d \omega) e^{-i \omega}, \\
e^{\hat{2}} & =\sqrt{k}(d \rho+i \operatorname{coth} \rho d \omega) e^{i \omega}, \\
e^{\hat{3}} & =\sqrt{k}(d \theta-i \tan \theta d \varphi) e^{-i \varphi}+\epsilon \frac{e^{-n i(\omega-\varphi)} \sin ^{n-2} \theta}{\cosh ^{n} \rho} \sqrt{k}(d \theta+i \tan \theta d \varphi) e^{-i \varphi}, \\
e^{\hat{4}} & =\sqrt{k}(d \theta+i \tan \theta d \varphi) e^{i \varphi}+\epsilon \frac{e^{n i(\omega-\varphi)} \sin ^{n-2} \theta}{\cosh ^{n} \rho} \sqrt{k}(d \theta-i \tan \theta d \varphi) e^{i \varphi} .
\end{aligned}
$$

We also define for convenience the unperturbed vielbeins in the compact directions

$$
\begin{aligned}
& e_{0}^{\hat{3}}=\sqrt{k}(d \theta-i \tan \theta d \varphi) e^{-i \varphi}, \\
& e_{0}^{\hat{4}}=\sqrt{k}(d \theta+i \tan \theta d \varphi) e^{i \varphi}
\end{aligned}
$$

The connection one-form $\omega_{\hat{\jmath}}^{\hat{\imath}}=\Gamma_{\hat{k} \hat{\jmath}}^{\hat{\imath}} e^{\hat{k}}$ is defined as usual by

$$
d e^{\hat{\imath}}+\omega_{\hat{\jmath}}^{\hat{\imath}} \wedge e^{\hat{\jmath}}=0
$$


and the curvature two-form

$$
R_{\hat{\jmath}}^{\hat{\imath}}=d \omega_{\hat{\jmath}}^{\hat{\imath}}+\omega_{\hat{k}}^{\hat{\imath}} \wedge \omega_{\hat{\jmath}}^{\hat{k}}=\frac{1}{2} R_{\hat{\jmath} \hat{k} \hat{l}}^{\hat{\imath}} e^{\hat{k}} \wedge e^{\hat{l}} .
$$

The non-vanishing components of the connection and curvature forms corresponding to (D.2) read

$$
\begin{aligned}
\sqrt{k} \omega_{\hat{1}}^{\hat{1}}=-\sqrt{k} \omega_{\hat{2}}^{\hat{2}}= & -\frac{\operatorname{coth} \rho}{2}\left[e^{i \omega} e^{\hat{1}}-e^{-i \omega} e^{\hat{2}}\right] \\
\sqrt{k} \omega_{\hat{3}}^{\hat{3}}=-\sqrt{k} \omega_{\hat{4}}^{\hat{4}}= & \frac{\tan \theta}{2}\left[e^{i \varphi} e^{\hat{3}}-e^{-i \varphi} e^{\hat{4}}\right] \\
& +\frac{\epsilon}{2} \frac{\tan \theta \sin ^{n-2} \theta}{\cosh ^{n} \rho}\left[-e^{n i(\omega-\varphi)+i \varphi} e^{\hat{3}}+e^{-n i(\omega-\varphi)-i \varphi} e^{\hat{4}}\right], \\
\sqrt{k} \omega_{\hat{4}}^{\hat{1}}=-\sqrt{k} \omega_{\hat{2}}^{\hat{3}}= & \epsilon n \frac{\tanh \rho \sin ^{n-2} \theta}{\cosh ^{n} \rho} e^{-n i(\omega-\varphi)-2 i \varphi-i \omega} e^{\hat{4}} \\
\sqrt{k} \omega_{\hat{3}}^{\hat{2}}=-\sqrt{k} \omega_{\hat{1}}^{\hat{4}}= & \epsilon n \frac{\tanh \rho \sin ^{n-2} \theta}{\cosh ^{n} \rho} e^{n i(\omega-\varphi)+2 i \varphi+i \omega} e^{\hat{3}}
\end{aligned}
$$

and

$$
\begin{aligned}
k R_{\hat{1}}^{\hat{1}}=-k R_{\hat{2}}^{\hat{2}}= & -\frac{1}{\sinh ^{2} \rho} e^{\hat{1}} \wedge e^{\hat{2}} \\
k R_{\hat{3}}^{\hat{3}}=-k R_{\hat{4}}^{\hat{4}}= & -\frac{1}{\cos ^{2} \theta} e^{\hat{3}} \wedge e^{\hat{4}} \\
& +\epsilon n \frac{\tan \theta \sin ^{n-2} \theta \tanh \rho}{\cosh ^{n} \rho} e^{n i(\omega-\varphi)+i \varphi+i \omega} e^{\hat{1}} \wedge e^{\hat{3}} \\
& -\epsilon n \frac{\tan \theta \sin ^{n-2} \theta \tanh \rho}{\cosh ^{n} \rho} e^{-n i(\omega-\varphi)-i \varphi-i \omega} e^{\hat{2}} \wedge e^{\hat{4}} \\
& +2 \epsilon \frac{\tan ^{2} \theta \sin ^{n-2} \theta}{\cosh ^{n} \rho} \cos n(\omega-\varphi) e^{\hat{3}} \wedge e^{\hat{4}}, \\
k R_{\hat{4}}^{\hat{1}}=-k R_{\hat{2}}^{\hat{3}}= & \epsilon n \frac{\left(1-n \sinh ^{2} \rho\right) \sin ^{n-2} \theta}{\cosh ^{n+2} \rho} e^{-n i(\omega-\varphi)-2 i \varphi-2 i \omega} e^{\hat{2}} \wedge e^{\hat{4}} \\
& +\epsilon n \frac{\tan \theta \sin ^{n-2} \theta \tanh \rho}{\cosh ^{n} \rho} e^{-n i(\omega-\varphi)-i \varphi-i \omega} e^{\hat{3}} \wedge e^{\hat{4}} \\
k R_{\hat{3}}^{\hat{2}}=-k R_{\hat{1}}^{\hat{4}}= & \epsilon n \frac{\left(1-n \sinh ^{2} \rho\right) \sin ^{n-2} \theta}{\cosh ^{n+2} \rho} e^{n i(\omega-\varphi)+2 i \varphi+2 i \omega} e^{\hat{1}} \wedge e^{\hat{3}} \\
& -\epsilon n \frac{\tan \theta \sin ^{n-2} \theta \tanh \rho}{\cosh ^{n} \rho} e^{n i(\omega-\varphi)+i \varphi+i \omega} e^{\hat{3}} \wedge e^{\hat{4}} .
\end{aligned}
$$

\section{References}

[1] R.C. Myers, "New dimensions for old strings," Phys. Lett. 199B (1987) 371.

[2] I. Antoniadis, C. Bachas, J.R. Ellis and D.V. Nanopoulos, "An Expanding Universe in String Theory," Nucl. Phys. B328 (1989) 117. 
[3] C.G. Callan, J.A. Harvey and A. Strominger, "World sheet approach to heterotic instantons and solitons," Nucl. Phys. B359 (1991) 611.

[4] M.J. Duff and J. X. Lu, "Elementary five-brane solutions of D $D 10$ supergravity," Nucl. Phys. B354 (1991) 141.

[5] C. Kounnas, M. Porrati and B. Rostand, "On $\mathcal{N}=4$ extended superliouville theory," Phys. Lett. 258B (1991) 61.

[6] K. Sfetsos, "Branes for Higgs phases and exact conformal field theories," JHEP 9901 (1999) 015 [arXiv:hep-th/9811167].

[7] O. Aharony, M. Berkooz, D. Kutasov and N. Seiberg, "Linear dilatons, NS5-branes and holography,” JHEP 9810 (1998) 004 [arXiv:hep-th/9808149].

[8] O. Aharony, B. Fiol, D. Kutasov and D.A. Sahakyan, "Little string theory and heterotic/type II duality," Nucl. Phys. B679 (2004) 3 [arXiv:hep-th/0310197].

[9] O. Aharony, A. Giveon and D. Kutasov, "LSZ in LST," Nucl. Phys. B691 (2004) 3 [arXiv:hep-th/0404016].

[10] E. Kiritsis, C. Kounnas, P.M. Petropoulos and J. Rizos, "Five-brane configurations without a strong-coupling regime," Nucl. Phys. B652, 165 (2003) [arXiv:hep-th/0204201].

[11] C. Bachas, A. Fotopoulos, P.M. Petropoulos and S. Ribault, unpublished work, 2003.

[12] D. Israël, C. Kounnas and P.M. Petropoulos, "Superstrings on NS5 backgrounds, deformed $A d S_{3}$ and holography," JHEP 0310 (2003) 028 [arXiv:hep-th/0306053].

[13] E. Kiritsis, C. Kounnas, P.M. Petropoulos and J. Rizos, "Five-brane configurations, conformal field theories and the strong-coupling problem,", Corfu 2001 Proceedings, arXiv:hep-th/0312300.

[14] D. Israël, C. Kounnas, A. Pakman and J. Troost, "The partition function of the supersymmetric two-dimensional black hole and little string theory," JHEP 0406 (2004) 033 [arXiv:hep-th/0403237].

[15] P.M. Petropoulos, "Deformations and geometric cosets," Fortschr. Phys. 53 (2005) 970. [arXiv:hep-th/0412328].

[16] S. Chaudhuri and J.A. Schwartz, "A criterion for integrably marginal oporators", Phys. Lett. 219B (1989) 291.

[17] P.M. Petropoulos and K. Sfetsos, "NS5-branes on an ellipsis and novel marginal deformations with parafermions," JHEP 0601 (2006) 167 [arXiv:hep-th/0512251].

[18] Z.A. Qiu, "Non-local current algebra and $\mathcal{N}=2$ superconformal field theory in two-dimensions," Phys. Lett. 188B (1987) 207. 
[19] V.A. Fateev and A.B. Zamolodchikov, "Parafermionic currents in the two-dimensional conformal quantum field theory and selfdual critical points in $Z(N)$ invariant statistical systems," Sov. Phys. JETP 62, 215 (1985) [Zh. Eksp. Teor. Fiz. 89, 380 (1985)].

[20] J.D. Lykken, "Finitely reducible realizations of the $\mathcal{N}=2$ superconformal algebra," Nucl. Phys. B313, 473 (1989).

[21] D. Karabali, Q.H. Park, H.J. Schnitzer and Z. Yang, "A GKO construction based on a path-integral formulation of gauged Wess-Zumino-Witten actions," Phys. Lett. 216B (1989) 307.

[22] K. Bardacki, M.J. Crescimanno and E. Rabinovici, "Parafermions from coset models," Nucl. Phys. B344 (1990) 344.

[23] E. Witten, "On string theory and black holes," Phys. Rev. D44 (1991) 314.

[24] N.J. Vilenkin, Special Functions and the Theory of Group Representions, Amer. Math. Soc, Providence, R.I., 1968.

[25] S. Chaudhuri and J.D. Lykken, "String theory, black holes, and $S L(2, \mathbb{R})$ current algebra," Nucl. Phys. B396 (1993) 270 [arXiv:hep-th/9206107].

[26] J.M. Maldacena, "The large-N limit of superconformal field theories and supergravity," Adv. Theor. Math. Phys. 2 (1998) 231 [Int. J. Theor. Phys. 38 (1999) 1113] [arXiv:hep-th/9711200].

[27] S.S. Gubser, I.R. Klebanov and A.M. Polyakov, "Gauge theory correlators from non-critical string theory," Phys. Lett. B428 (1998) 105 [arXiv:hep-th/9802109].

[28] E. Witten, “Anti-de Sitter space and holography," Adv. Theor. Math. Phys. 2 (1998) 253 [arXiv:hep-th/9802150].

[29] I.R. Klebanov and E. Witten, "AdS/CFT correspondence and symmetry breaking," Nucl. Phys. B556 (1999) 89 [arXiv:hep-th/9905104].

[30] W. Lerche, C. Vafa and N.P. Warner, "Chiral rings in $\mathcal{N}=2$ superconformal theories," Nucl. Phys. B324 (1989) 427.

[31] D.A. Sahakyan and T. Takayanagi, "On the connection between $N=2$ minimal string and $(1, n)$ bosonic minimal string," JHEP 0606 (2006) 027 [arXiv:hep-th/0512112].

[32] V. Niarchos, "On minimal $N=4$ topological strings and the $(1, k)$ minimal bosonic string," JHEP 0603 (2006) 045 [arXiv:hep-th/0512222].

[33] A. Giveon, D. Kutasov and O. Pelc, "Holography for non-critical superstrings," JHEP 9910 (1999) 035 [arXiv:hep-th/9907178].

[34] B. Zumino, "Supersymmetry and Kähler manifolds," Phys. Lett. B87 (1979) 203. 
[35] L. Alvarez-Gaume and D.Z. Freedman, "Geometrical structure and ultraviolet finiteness in the supersymmetric sigma model," Commun. Math. Phys. 80 (1981) 443.

[36] D.Z. Freedman and P.K. Townsend, "Antisymmetric tensor gauge theories and non-linear sigma models," Nucl. Phys. B177 (1981) 282.

[37] P.S. Howe and G. Sierra, "Two-dimensional supersymmetric non-linear sigma models with torsion," Phys. Lett. 148B (1984) 451.

[38] J. Bagger, D. Nemeschansky, N. Seiberg and S. Yankielowicz, "Bosons, fermions and Thirring strings," Nucl. Phys. B289, 53 (1987).

[39] C. Kounnas, "Four-dimensional gravitational backgrounds based on $\mathcal{N}=4, c=4$ superconformal systems," Phys. Lett. 321B (1994) 26 [arXiv:hep-th/9304102].

[40] M. Ademollo et al., "Dual string models with non-abelian color and flavor symmetries," Nucl. Phys. B114 (1976) 297.

[41] N. Berkovits and C. Vafa, “N=4 topological strings,” Nucl. Phys. B433 (1995) 123 [arXiv:hep-th/9407190].

[42] I. Bakas and E. Kiritsis, "Beyond the large- $N$ limit: non-linear $W(\infty)$ as symmetry of the $S L(2, \mathbb{R}) / U(1)$ coset model," Int. J. Mod. Phys. 751A (1992) 55 [Int. J. Mod. Phys. 7A (1992) 55] [arXiv:hep-th/9109029]. 